Draft Version November 16, 2018

Preprint typeset using $\mathrm{LAT}_{\mathrm{EX}}$ style emulateapj v. 26/01/00

\title{
A GENERAL FORMULATION OF THE SOURCE CONFUSION STATISTICS AND APPLICATION TO INFRARED GALAXY SURVEYS
}

\author{
Tsutomu T. Takeuchi ${ }^{1,3}$ \\ AND \\ TAKAKO T. IsHII ${ }^{2,3}$ \\ Draft version November 16, 2018
}

\begin{abstract}
Source confusion has been a long-standing problem in the astronomical history. In the previous formulation of the confusion problem, sources are assumed to be distributed homogeneously on the sky. This fundamental assumption is, however, not realistic in many applications. In this work, by making use of the point field theory, we derive general analytic formulae for the confusion problems with arbitrary distribution and correlation functions. As a typical example, we apply these new formulae to the source confusion of infrared galaxies. We first calculate the confusion statistics for power-law galaxy number counts as a test case. When the slope of differential number counts, $\gamma$, is steep, the confusion limits becomes much brighter and the probability distribution function (PDF) of the fluctuation field is strongly distorted. Then we estimate the PDF and confusion limits based on the realistic number count model for infrared galaxies. The gradual flattening of the slope of the source counts makes the clustering effect rather mild. Clustering effects result in an increase of the limiting flux density with $\sim 10 \%$. In this case, the peak probability of the PDF decreases up to $\sim 15 \%$ and its tail becomes heavier. Though the effects are relatively small, they will be strong enough to affect the estimation of galaxy evolution from number count or fluctuation statistics. We also comment on future submillimeter observations.
\end{abstract}

Subject headings: methods: statistical — galaxies: statistics — infrared: galaxies — large-scale structure of universe - submillimeter — surveys

\section{INTRODUCTION}

Astronomical source counts often suffer from a problem that multiple sources are located in a single beam of the observational instrument. The obtained number counts will then be different from true ones, because the apparent position and flux of the sources are changed by blending of other, usually fainter sources. This is called the source confusion, and the resulting measurement error is referred to as the confusion noise.

In its early stage, confusion problem has been investigated and formulated mainly by radio astronomers in relation to the so-called fluctuation analysis or $P(D)$ analysis (Scheuer 1957; Hewish 1961; Bennett 1962; Murdoch et al. 1973; Condon 1974; Condon \& Dressel 1978; Wall 1978; Wall et al. 1982). This analysis has been immediately applied and developed further in X-ray (e.g., Scheuer 1974; Franceschini 1982; Barcons \& Fabian 1990; Barcons 1992; Barcons et al. 1994) and infrared astronomy (e.g., Hacking \& Houck 1987; Franceschini et al. 1989; Oliver et al. 1997; Matsuhara et al. 2000; Lagache \& Puget 2000; Miville-Deschênes, Lagache, \& Puget 2002; Friedmann \& Bouchet 2003). Now confusion problem also becomes important in submillimeter cosmology and high-precision astrometric missions (cf. Hogg 2001).

Cosmologists compare model predictions with observed source counts to extract information of the evolution of galaxies and other objects (e.g., Franceschini et al. 1991; Guiderdoni et al. 1998; Takeuchi et al. 1999; Hirashita et al. 1999; Franceschini et al. 2001; Takeuchi et al. 2001a,b). The confusion noise often prevents us from direct comparison between measured counts and predictions. Especially, recent advent of infrared (IR) and submillimeter facilities may have stimulated the discussion on the confusion problem. Compared with other wavelengths, we have relatively small aperture telescopes in the IR, mainly because ground-based observations are almost impossible, and we inevitably need cooled space telescopes. In the submillimeter the antenna is relatively large up to $\sim 10-30 \mathrm{~m}$, but the long wavelength also makes the single-dish surveys confusion-limited by a large diffraction.

Generally, the measurement error of the source flux blurs the true source counts. This problem was originally pointed out by Eddington (1913) and deeply considered in subsequent studies (e.g., Eddington 1940; Bennett 1962; Refsdal 1969; Murdoch et al. 1973; Hogg \& Turner 1998), but there still remains some unsolved issues, especially when the error is dominated by the confusion noise. Recently Monte Carlo simulation has become popular to evaluate confusion effect (e.g., Bertin et al. 1997; Eales et al. 2000; Scott et al. 2002), but such an approach is sometimes not easy to interpret, and often time consuming. Today, many large survey projects will be performed or completed soon, and a general analytical prescription for the confusion is desirable.

Fundamental assumption for the formulation of the confusion problem is that the sources are distributed homogeneously on the sky (e.g., Scheuer 1957; Condon 1974; Franceschini et al. 1989). However, this assumption is obviously not realistic in many applications, e.g., stars in the Galaxy, or galaxies in the Universe. A straightforward attempt to take into account

\footnotetext{
${ }^{1}$ National Astronomical Observatory of Japan, Mitaka, Tokyo 181-8588, JAPAN; takeuchi@optik.mtk.nao.ac.jp.

${ }^{2}$ Kwasan and Hida Observatories, Kyoto University, Yamashina-ku, Kyoto 607-8471, JAPAN; ishii@kwasan.kyoto-u.ac.jp.

${ }^{3}$ Research Fellows of the Japan Society for the Promotion of Science
} 
the source clustering is to integrate over the spatial correlation functions along the line of sight. This approach has been adopted by several works (e.g., Barcons \& Fabian 1988; Barcons, Fabian, \& Carrera 1998; Burigana \& Popa 1998). Among them, Toffolatti et al. (1998) presented comprehensive results closely related to the present issue. Since it requires the knowledge of three-dimensional correlation functions, while it is a convenient method for theoretical predictions, observationally it is quite rare that we can obtain spatial information at the early phase of a survey. Hence, we need a method to evaluate the confusion only from the projected information of source clustering. In this line, Barcons (1992) has pioneered the methodology to tackle the riddle, but his results were restricted to a few simple cases. Since then, little analytical progress on this topic has been made up to now.

In this work, by making extensive use of the methods of the theory of point process, we show the general analytic formula for the confusion problems with arbitrary distribution and correlation functions. In Section 2, we first consider the fluctuation of unclustered sources, and then present the general formulae for inhomogeneous and clustered source distributions. Based on the new formulae, we reconsider how to treat the confusion noise in Section 3. In Section 4 we formulate higher-order clustering of galaxies, which will be included in the general confusion problems. We focus on far-infrared and submillimeter galaxies. Section 5 is devoted to our conclusions. Factorial moments and cumulants are introduced in Appendix A. In Appendix B we derive the first- and second-order cumulants of clustered field. We present a general expression for the rule-of-thumb to avoid confusion in Appendix C. Glossary of the symbols are provided in Appendix D.

\section{STATISTICS OF THE FLUCTUATION FIELD}

Statistics of fluctuation field is a fundamental tool for the confusion problem. We first formulate the fluctuation of confusion noise in line with classical works (Scheuer 1957, 1974), and then we translate it to the language of the theory of point fields (Daley \& Vere-Jones 2003). By this method, we can straightforwardly extend our formulation to the confusion problem of clustered sources (cf. Barcons 1992).

\subsection{Fluctuation Generated from Sources without Clustering}

\subsubsection{Classical derivation}

We define a probability density function (hereafter PDF) of a source having a flux $S^{\prime} \in[S, S+d S], \mathfrak{p}(S) d S$, i.e.,

$$
\int_{0}^{\infty} \mathfrak{p}(S) d S=1
$$

We set $h(\boldsymbol{x})=h(\theta, \phi)$, the beam pattern (normalized to unity at the beam center), and $s(\boldsymbol{x})=S h(\boldsymbol{x})$, the system response from the intrinsic flux $S$.

The total signal response we observe, $I(\boldsymbol{x})$, is described as

$$
I(\boldsymbol{x})=\sum_{n=0}^{\infty} S_{n} h\left(\boldsymbol{x}-\boldsymbol{x}_{n}\right),
$$

where $S_{n}$ is the flux of the $n$-th source, and $\boldsymbol{x}_{n}$ is the angular position of the source. For the following discussion we need to define a flux signal that consists of exactly $N$ sources, $I_{N}(\boldsymbol{x})$,

$$
I_{N}(\boldsymbol{x})=\sum_{n=0}^{N} S_{n} h\left(\boldsymbol{x}-\boldsymbol{x}_{n}\right)
$$

Consider an ensemble of the number of sources in a unit solid angle, $n_{\ell}, \ell=1,2, \ldots$, and let the mean of $n_{\ell}$ is $\bar{n}$. Here, $\mathfrak{N}(S) \equiv \bar{n} \mathfrak{p}(S)$ gives the familiar differential number count of the considered sources. Then, from the no clustering assumption, the probability of observing exactly $N$ sources in a beam is given by the Poisson process

$$
p_{N}=\frac{\left(\bar{n} \Omega_{\mathrm{b}}\right)^{N}}{N !} e^{-\bar{n} \Omega_{\mathrm{b}}}
$$

where $\Omega_{\mathrm{b}}$ is the solid angle of the beam.

Then let us consider the PDF of the value of $I(\boldsymbol{x}), f(I),{ }^{4}$

$$
\begin{aligned}
f(I) & =\operatorname{Prob}\left\{I^{\prime} \in[I, I+d I)\right\} \\
& =\sum_{N=0}^{\infty} \operatorname{Prob}\{\text { exactly } N \text { sources lie in a beam }\} \operatorname{Prob}\left\{I_{N}(\boldsymbol{x}) \in[I, I+d I)\right\} \\
& \equiv \sum_{N=0}^{\infty} p_{N} g_{N}(I),
\end{aligned}
$$

where $g_{N}(I)$ denotes the PDF of $I_{N}(t)$.

\footnotetext{
${ }^{4}$ This function corresponds to $P(D)$ in radioastronomical terminology.
} 
Under a certain regularity condition, a PDF is uniquely characterized via its Laplace transform (LT) $)^{5}$ We define the LTs of $f(I)$ and $g_{N}(I), \mathcal{L}_{f}(t)$ and $\mathcal{L}_{g_{N}}(t)$, respectively,

$$
\begin{aligned}
& \mathcal{L}_{f}(t) \equiv \mathrm{E}\left[e^{-t I}\right]=\int_{-\infty}^{\infty} e^{-t I} f(I) d I, \\
& \mathcal{L}_{g_{N}}(t)=\int_{-\infty}^{\infty} e^{-t I} g_{N}(I) d I
\end{aligned}
$$

where $\mathrm{E}[\cdot]$ represents the expectation value of a random variable. Then we obtain

$$
\mathcal{L}_{f}(t)=\sum_{N=0}^{\infty} p_{N} \mathcal{L}_{g_{N}}(t) .
$$

Hence, concrete expression of $\mathcal{L}_{g_{N}}(t)$ is our next step to have the functional form of $f(I)$.

We consider random variables $s_{n}=S_{n} h\left(\boldsymbol{x}-\boldsymbol{x}^{\prime}\right)$ and their summation over $n, I_{N}(\boldsymbol{x})=\sum_{n=0}^{N} s_{n}$. Then the PDF of a signal $I$ with $N$ summands is represented by the following recursive convolution:

$$
\begin{aligned}
g_{N+1}(I) & =\int_{0}^{I} g_{N}\left(I-s^{\prime}\right) g\left(s^{\prime}\right) d s^{\prime}, \\
g_{1}(I) & =g(I) .
\end{aligned}
$$

Since the LT of a convolution of two functions is a normal product of the LTs of them, the PDF of $s$,

$$
g(s) \equiv \operatorname{Prob}\left\{s^{\prime} \in[s, s+d s)\right\}
$$

gives

$$
\mathcal{L}_{g_{N}}(t)=\mathcal{L}_{g}(t)^{N}
$$

where

$$
\mathcal{L}_{g}(t)=\int_{-\infty}^{\infty} e^{-t s} g(s) d s
$$

Then we have

$$
\begin{aligned}
\mathcal{L}_{f}(t) & =\sum_{N=0}^{\infty} \frac{\left(\bar{n} \Omega_{\mathrm{b}}\right)^{N} e^{-\bar{n} \Omega_{\mathrm{b}}}}{N !} \mathcal{L}_{g}(t)^{N} \\
& =e^{-\bar{n} \Omega_{\mathrm{b}}} \sum_{N=0}^{\infty} \frac{\left(\bar{n} \Omega_{\mathrm{b}}\right)^{N} \mathcal{L}_{g}(t)^{N}}{N !} \\
& =e^{-\bar{n} \Omega_{\mathrm{b}}} e^{\bar{n} \Omega_{\mathrm{b}} \mathcal{L}_{g}(t)}=e^{\bar{n} \Omega_{\mathrm{b}}\left[\mathcal{L}_{g}(t)-1\right]}
\end{aligned}
$$

The rest we should consider is the exact form of $\mathcal{L}_{g}(t)$. The profile of a single source is expressed as $s(\boldsymbol{x})=\operatorname{Sh}(\boldsymbol{x})$. Since the LT of a random variable $s$ is a statistical average of $e^{-t s}$, we obtain

$$
\mathcal{L}_{g}(t)=\frac{1}{\Omega_{\mathrm{b}}} \int_{\Omega_{\mathrm{b}}} \int_{S} e^{-t S h(\boldsymbol{x})} \mathfrak{p}(S) d S d \boldsymbol{x}
$$

Here we used a simplified symbol $\int_{S} \equiv \int_{S=0}^{\infty}$, i.e., integration with respect to $S$ over the range of $[0, \infty]$. We use this notation for some other variables in the following.

Substituting Equation (15) into Equation (14), we finally obtain

$$
\begin{aligned}
f(I) & =\mathcal{L}^{-1}\left[e^{\bar{n} \Omega_{\mathrm{b}}\left[\mathcal{L}_{g}(t)-1\right]}\right] \\
& =\mathcal{L}^{-1}\left[\exp \bar{n} \int_{\Omega_{\mathrm{b}}}\left[\int_{S} e^{-t S h(\boldsymbol{x})} \mathfrak{p}(S) d S-1\right] d \boldsymbol{x}\right],
\end{aligned}
$$

where $\mathcal{L}^{-1}[\cdot]$ represents the inverse Laplace transform. By the formula for moments

$$
\mu_{k} \equiv \mathrm{E}\left[I^{k}\right]=\left.(-1)^{k} \frac{d^{k} \mathcal{L}_{f}(t)}{d t^{k}}\right|_{t=0},
$$

and cumulants (reduced moments)

$$
\kappa_{k}=\left.(-1)^{k} \frac{d^{k} \ln \mathcal{L}_{f}(t)}{d t^{k}}\right|_{t=0},
$$

${ }^{5}$ If we use $i t$ instead of $t$, we obtain a characteristic function $(\mathrm{CF})$. While the CF is also quite common in physical studies, here we use LT to refer literatures in mathematics and statistics. 
(e.g., Stuart \& Ord 1994) we obtain the following beautiful expressions for the cumulants of $I$ (the simplest result of Campbell's theorem: Campbell 1909; Rice 1944).

$$
\begin{aligned}
& \kappa_{1}=\mathrm{E}[I]=\mu_{1}=\bar{n} \int_{\Omega_{\mathrm{b}}} \int_{S} S h(\boldsymbol{x}) \mathfrak{p}(S) d S d \boldsymbol{x}=\int_{\Omega_{\mathrm{b}}} \int_{S} S h(\boldsymbol{x}) \mathfrak{N}(S) d S d \boldsymbol{x}, \\
& \kappa_{2}=\mathrm{E}\left[\left(I-\kappa_{1}\right)^{2}\right]=\int_{\Omega_{\mathrm{b}}} \int_{S} S^{2} h(\boldsymbol{x})^{2} \mathfrak{N}(S) d S d \boldsymbol{x}, \ldots,
\end{aligned}
$$

and generally,

$$
\kappa_{k}=\int_{\Omega_{\mathrm{b}}} \int_{S} S^{k} h(\boldsymbol{x})^{k} \mathfrak{N}(S) d S d \boldsymbol{x} .
$$

\subsubsection{Alternative derivation via point field theory}

The position of galaxies, stars, or other point sources is expressed as a point in the field under consideration. A mathematical technique to treat such a filed of points is called the theory of point process, and has a long history (e.g., Stoyan \& Stoyan 1994; Stoyan, Kendall, \& Mecke 1995; Daley \& Vere-Jones 2003). The theory provides us very powerful tools for the problems that we consider here. Thus, we translate the above heuristic derivation of $f(I)$ with the language of point process theory. Considering the problem along this line enables us a straightforward extension of our discussion to the case of clustered sources.

The fluctuation filed $I(\boldsymbol{x})$ is expressed as

$$
\begin{aligned}
I(\boldsymbol{x}) & =\sum_{n=0}^{\infty} S_{n} h\left(\boldsymbol{x}-\boldsymbol{x}_{n}\right) \\
& =\int_{\mathbb{R}^{2}} h\left(\boldsymbol{x}-\boldsymbol{x}^{\prime}\right) S\left(\boldsymbol{x}^{\prime}\right) \mathcal{N}\left(d \boldsymbol{x}^{\prime}\right),
\end{aligned}
$$

where $\mathcal{N}(A)$ of Borel sets $A \in \mathbb{R}^{2}$ is the so-called 'counting measure', which represents the number of points in the set $A$, and $S\left(\boldsymbol{x}^{\prime}\right)$ is a fictitious stochastic process that takes a value $S\left(\boldsymbol{x}_{n}^{\prime}\right)=S_{n}$ at each point $\boldsymbol{x}_{n}^{\prime}$ (Daley \& Vere-Jones 2003). Here we identify the celestial sphere with a real plane $\mathbb{R}^{2}$. We define a random measure $\mathcal{M}(A)$ as

$$
\begin{aligned}
\mathcal{M}(A) & \equiv \int_{A} I(\boldsymbol{x}) d \boldsymbol{x} \\
& =\int_{A}\left[\int_{\mathbb{R}^{2}} h\left(\boldsymbol{x}-\boldsymbol{x}^{\prime}\right) S\left(\boldsymbol{x}^{\prime}\right) \mathcal{N}\left(d \boldsymbol{x}^{\prime}\right)\right] d \boldsymbol{x} \\
& =\sum_{\boldsymbol{x}_{n} \in A} S_{n} \int_{\mathbb{R}^{2}} h\left(\boldsymbol{x}-\boldsymbol{x}_{n}\right) d \boldsymbol{x} .
\end{aligned}
$$

Here we introduce a Laplace functional $\mathcal{L}_{\mathcal{M}}[\mathcal{X}]$ with respect to the random measure $\mathcal{M}$,

$$
\begin{aligned}
\mathcal{L}_{\mathcal{M}}[\mathcal{X}] & \equiv \mathrm{E}\left[\exp \left[-\int_{\mathbb{R}^{2}} \mathcal{X}(\boldsymbol{x}) \mathcal{M}(d \boldsymbol{x})\right]\right] \\
& =\mathrm{E}\left[\exp \left[-\int_{\mathbb{R}^{2}} \mathcal{X}(\boldsymbol{x}) I(\boldsymbol{x}) d \boldsymbol{x}\right]\right] .
\end{aligned}
$$

It is a Laplace counterpart of the characteristic functional of a random field, both of which are often used in particle physics, turbulence theory, and structure formation theories in the Universe, etc. (e.g., Vlad et al. 1994; Frisch 1995; Szapudi \& Szalay 1993; Matsubara 1995) ${ }^{6}$. We observe

$$
\begin{aligned}
\int_{\mathbb{R}^{2}} \mathcal{X}(\boldsymbol{x}) \mathcal{M}(d \boldsymbol{x}) & =\int_{\mathbb{R}^{2}} \mathcal{X}(\boldsymbol{x})\left[\sum_{n} S_{n} h\left(\boldsymbol{x}-\boldsymbol{x}_{n}\right)\right] d \boldsymbol{x} \\
& =\sum_{n} S_{n} \int_{\mathbb{R}^{2}} \mathcal{X}(\boldsymbol{x}) h\left(\boldsymbol{x}-\boldsymbol{x}_{n}\right) d \boldsymbol{x} .
\end{aligned}
$$

Here,

$$
\mathrm{E}\left[\exp \left[-S_{n} \int_{\mathbb{R}^{2}} \mathcal{X}(\boldsymbol{x}) h\left(\boldsymbol{x}-\boldsymbol{x}_{n}\right) d \boldsymbol{x}\right]\right]=\mathcal{L}_{\mathfrak{N}}\left[\int_{\mathbb{R}^{2}} \mathcal{X}(\boldsymbol{x}) h\left(\boldsymbol{x}-\boldsymbol{x}_{n}\right) d \boldsymbol{x}\right] \equiv \mathcal{Z}\left(\boldsymbol{x}_{n}\right)
$$

${ }^{6}$ In statistical and particle physics, $\mathcal{L}_{\mathcal{M}}[\mathcal{X}]$ is often expressed in the form

$$
\mathcal{L}_{\mathcal{M}}[\mathcal{X}]=\int \mathfrak{D}[I] \mathfrak{F}[I] e^{-\int \mathcal{X}(\boldsymbol{x}) I(\boldsymbol{x}) d \boldsymbol{x}},
$$

in relation to the path integral formulation (e.g., Fry 1984a; Szapudi \& Szalay 1993; Matsubara 1995). Here $\mathfrak{F}[I]$ is the assigned probability functional for an overall configuration of the field $I(\boldsymbol{x})$. 
In Equation $(26), \mathcal{L}_{\mathfrak{N}}[\cdot]$ represents the Laplace-Stieltjes functional with respect to the PDF of $S_{n}, \mathfrak{p}\left(S_{n}\right)$, i.e.,

$$
\mathcal{L}_{\mathfrak{N}}\left[\int_{\mathbb{R}^{2}} \mathcal{X}(\boldsymbol{x}) h\left(\boldsymbol{x}-\boldsymbol{x}_{n}\right) d \boldsymbol{x}\right] \equiv \int_{S} \exp \left[-S_{n} \int_{\mathbb{R}^{2}} \mathcal{X}(\boldsymbol{x}) h\left(\boldsymbol{x}-\boldsymbol{x}_{n}\right) d \boldsymbol{x}\right] \mathfrak{p}\left(S_{n}\right) d S_{n} .
$$

Combining Equations (24), (25), and (26), we have an important relation

$$
\begin{aligned}
\mathcal{L}_{\mathcal{M}}[\mathcal{X}] & =\prod_{n} \mathrm{E}\left[\exp \left[-S_{n} \int_{\mathbb{R}^{2}} \mathcal{X}(\boldsymbol{x}) h\left(\boldsymbol{x}-\boldsymbol{x}_{n}\right) d \boldsymbol{x}\right]\right] \\
& =\prod_{n} \mathcal{Z}\left(\boldsymbol{x}_{n}\right) \\
& =G[\mathcal{Z}] .
\end{aligned}
$$

The last step is the definition of the probability generating functional (PGFL) of a point field, $G[\mathcal{Z}]$ (cf. Balian \& Schaeffer 1989; Daley \& Vere-Jones 2003). This shows an important fact: the fluctuation field blurred by a beam profile is expressed in terms of the PGFL of the original point field. To be exact, we can describe the observed fluctuation field as an explicit functional of the true point field.

In general, $G[\mathcal{Z}]$ has some useful expansions with respect to the factorial moments, factorial cumulants, and other related summary statistics of the point field (Vlad et al. 1994; Kerscher 2001; Daley \& Vere-Jones 2003). The most familiar statistic for astrophysical studies may be the correlation function (or equivalently, normalized factorial cumulants). Then, $G[\mathcal{Z}]$ can be expressed in the following form (for the proof, see, e.g., Ma 1985):

$$
\begin{aligned}
\ln G[\mathcal{Y}+1] & =\sum_{k=1}^{\infty} \frac{1}{k !} \int_{\mathbb{R}^{2} \times \cdots \times \mathbb{R}^{2}} \ldots \int_{k=1} \mathcal{Y}\left(\boldsymbol{x}_{1}\right) \cdots \mathcal{Y}\left(\boldsymbol{x}_{k}\right) c_{[k]} d \boldsymbol{x}_{1} \cdots d \boldsymbol{x}_{k} \\
& =\sum_{k=1}^{\infty} \frac{\bar{n}^{k}}{k !} \int_{\mathbb{R}^{2} \times \cdots \times \mathbb{R}^{2}} \mathcal{Y}\left(\boldsymbol{x}_{1}\right) \cdots \mathcal{Y}\left(\boldsymbol{x}_{k}\right) w_{k} d \boldsymbol{x}_{1} \cdots d \boldsymbol{x}_{k},
\end{aligned}
$$

where $c_{[k]}$ denotes the factorial cumulant of the point field (see Appendix A), and $w_{k}=w_{k}\left(\boldsymbol{x}_{1}, \cdots, \boldsymbol{x}_{k}\right)$ is the angular $k$-point correlation function of the point sources. This relation will be used to extend our formulation to the clustered point sources in $\S 2.3$. For a Poisson field, by its definition, all the higher order $(k \geq 2)$ factorial cumulants vanish, and we obtain

$$
\ln G[\mathcal{Y}+1]=\bar{n} \int_{\mathbb{R}^{2}} \mathcal{Y}(\boldsymbol{x}) d \boldsymbol{x} .
$$

Hence, by substituting $\mathcal{Z}=\mathcal{Y}+1$ in Equation (30) and doing some algebra, we obtain

$$
G[\mathcal{Z}]=e^{\bar{n} \int_{\mathbb{R}^{2}}[\mathcal{Z}(\boldsymbol{x})-1] d \boldsymbol{x}} .
$$

Now returning back to Equation (24),

$$
\mathcal{L}_{\mathcal{M}}[\mathcal{X}]=G[\mathcal{Z}]=e^{\bar{n} \int_{\mathbb{R}^{2}}[\mathcal{Z}(\boldsymbol{x})-1] d \boldsymbol{x}} .
$$

In order to obtain the formula for the local process in a beam area $\Omega_{\mathrm{b}}$, we set the test function $\mathcal{Z}(\boldsymbol{x})$ as

$$
\mathcal{Z}^{*}(\boldsymbol{x})=1-[1-\mathcal{Z}(\boldsymbol{x})] \mathbb{I}_{\Omega_{\mathrm{b}}},
$$

where $\mathbb{I}_{A}$ is an indicator function of a set $A$,

$$
\mathbb{I}_{A}= \begin{cases}1 & \boldsymbol{x} \in A, \\ 0 & \boldsymbol{x} \notin A .\end{cases}
$$

Substituting Equation (33) into Equation (32) yields

$$
\begin{aligned}
\mathcal{L}_{\mathcal{M}}[\mathcal{X}] & =e^{\bar{n} \int_{\mathbb{R}^{2}}\left[\mathcal{Z}^{*}(\boldsymbol{x})-1\right] d \boldsymbol{x}} \\
& =e^{\bar{n} \int_{\mathbb{R}^{2}}[\mathcal{Z}(\boldsymbol{x})-1] \mathbb{I}_{\Omega_{\mathrm{b}}} d \boldsymbol{x}} \\
& =e^{\bar{n} \int_{\Omega_{\mathrm{b}}}[\mathcal{Z}(\boldsymbol{x})-1] d \boldsymbol{x}} .
\end{aligned}
$$

Recall that

$$
\mathcal{Z}\left(\boldsymbol{x}_{n}\right)=\mathcal{L}_{\mathfrak{N}}\left[\int_{\mathbb{R}^{2}} \mathcal{X}(\boldsymbol{x}) h\left(\boldsymbol{x}-\boldsymbol{x}_{n}\right) d \boldsymbol{x}\right]
$$

we obtain

$$
\begin{aligned}
\mathcal{L}_{\mathcal{M}}[\mathcal{X}] & =\exp \bar{n} \int_{\Omega_{\mathrm{b}}}\left\{\mathcal{L}_{\mathfrak{N}}\left[\int_{\mathbb{R}^{2}} \mathcal{X}\left(\boldsymbol{x}^{\prime}\right) h\left(\boldsymbol{x}^{\prime}-\boldsymbol{x}\right) d \boldsymbol{x}^{\prime}\right]-1\right\} d \boldsymbol{x} \\
& =\exp \bar{n} \int_{\Omega_{\mathrm{b}}}\left\{\int_{S} e^{-S \int_{\mathbb{R}^{2}} \mathcal{X}\left(\boldsymbol{x}^{\prime}\right) h\left(\boldsymbol{x}^{\prime}-\boldsymbol{x}\right) d \boldsymbol{x}^{\prime}} \mathfrak{p}(S) d S-1\right\} d \boldsymbol{x}
\end{aligned}
$$


By setting the test function $\mathcal{X}\left(\boldsymbol{x}^{\prime}\right)=t$, we have

$$
\begin{aligned}
\mathcal{L}_{f}(t) & =\exp \bar{n} \int_{\Omega_{\mathrm{b}}}\left[\int_{S} e^{-t S h\left(\boldsymbol{x}^{\prime}-\boldsymbol{x}\right)} \mathfrak{p}(S) d S-1\right] d \boldsymbol{x} . \\
& =\exp \bar{n} \int_{\Omega_{\mathrm{b}}}\left[\int_{S} e^{-t S h(\boldsymbol{x})} \mathfrak{p}(S) d S-1\right] d \boldsymbol{x} .
\end{aligned}
$$

Inverting the Laplace transform, we again obtain the desired result

$$
f(I)=\mathcal{L}^{-1}\left[\exp \bar{n} \int_{\Omega_{\mathrm{b}}}\left[\int_{S} e^{-t S h(\boldsymbol{x})} \mathfrak{p}(S) d S-1\right] d \boldsymbol{x}\right],
$$

which is the same result with Equation (16).

\subsection{The Case of Inhomogeneous Poisson Point Field}

Sometimes we face a problem that the points distribute locally Poisson but the intensity $m$ is spatially inhomogeneous, i.e., depends on the position on the sky. The projected stellar density distribution of the Milky Way might be described in this way (e.g., Chandrasekhar \& Münch 1950).

If the typical angular scale of the variation of $\bar{n}$ is larger than the typical beam size, we only have to divide the sky into patches with the variation scale and derive the PDF of the fluctuation $f(I)$ in each patch by Equation (16).

On the other hand, the variation scale of $\bar{n}$ is comparable or smaller than the beam size, we should properly treat the variation within a beam. In such case $\bar{n}$ is expressed as $\bar{n}(\boldsymbol{x})$ at the scale of our interest. The intensity in a set $A, \bar{N}(A)$, is obtained by integrating $\bar{n}(\boldsymbol{x})$ over $A$, as

$$
\bar{N}(A)=\int_{A} \bar{n}(\boldsymbol{x}) d \boldsymbol{x},
$$

(Cressie 1993, pp.650-652). In this case the joint probability for some positions are still Poisson, and the independence still holds. It leads to the following formula

$$
\mathcal{L}_{f}(t)=\exp \int_{\Omega_{\mathrm{b}}}\left[\int_{S} e^{-t S h(\boldsymbol{x})} \mathfrak{p}(S) d S-1\right] \bar{n}(\boldsymbol{x}) d \boldsymbol{x} .
$$

The same as the above, the $\operatorname{PDF} f(I)$ is obtained by inverting the LT.

\subsection{Fluctuation Generated from Clustered Sources}

We now turn to the case of the point source with significant clustering. Clustering of the sources makes the confusion effect more severe, because the variance of the source number is larger than that of Poisson fluctuation. The importance of clustering in the fluctuation and confusion problems has already pointed out as early as the end of 1960's by Refsdal (1969), but he did not provided a quantitative answer in that paper. The fluctuation analysis of sky brightness including the effect of clustering was first presented by Barcons (1992) for the study of the X-ray background. The central tool for his analysis was characteristic functional of the field $I(\boldsymbol{x})$. We use its equivalent, Laplace functional here, and derive the formula in mathematically rigorous way, in parallel with the discussion in $\S 2.1 .2$.

Again we start with the fluctuation field $I(\boldsymbol{x})=\sum_{n=0}^{\infty} S_{n} h\left(\boldsymbol{x}-\boldsymbol{x}_{n}\right)$, but this time $\boldsymbol{x}_{n}$ are not distributed at random on the sky, but have a certain correlation with each other. The statistical properties of the field are characterized by $\mathcal{L}_{\mathcal{M}}[\mathcal{X}]$. Since the derivation of Equation (28) does not depend on the clustering property of the source point field, we can also apply Equations (28) and (29). Since the point field has non-vanishing correlation functions for clustered sources, Equation (29) reads

$$
\left.\ln G[\mathcal{Z}]=\sum_{k=1}^{\infty} \frac{\bar{n}^{k}}{k !} \int_{\mathbb{R}^{2} \times \cdots \times \mathbb{R}^{2}} \ldots \int_{\mathcal{Z}}\left[\boldsymbol{x}_{1}\right)-1\right] \cdots\left[\mathcal{Z}\left(\boldsymbol{x}_{k}\right)-1\right] w_{k}\left(\boldsymbol{x}_{1}, \cdots, \boldsymbol{x}_{k}\right) d \boldsymbol{x}_{1} \cdots d \boldsymbol{x}_{k},
$$

therefore

$$
G[\mathcal{Z}]=\exp \sum_{k=1}^{\infty} \frac{\bar{n}^{k}}{k !} \int_{\mathbb{R}^{2} \times \cdots \times \mathbb{R}^{2}}\left[\mathcal{Z}\left(\boldsymbol{x}_{1}\right)-1\right] \cdots\left[\mathcal{Z}\left(\boldsymbol{x}_{k}\right)-1\right] w_{k}\left(\boldsymbol{x}_{1}, \cdots, \boldsymbol{x}_{k}\right) d \boldsymbol{x}_{1} \cdots d \boldsymbol{x}_{k} .
$$

The same as the unclustered field, we set $\mathcal{Z}^{*}=1-(1-\mathcal{Z}) \mathbb{I}_{\Omega_{\mathrm{b}}}$, and obtain

$$
G[\mathcal{Z}]=\exp \sum_{k=1}^{\infty} \frac{\bar{n}^{k}}{k !} \int_{\Omega_{\mathrm{b}} \times \cdots \times \Omega_{\mathrm{b}}} \ldots \int_{\mathcal{Z}}\left[\mathcal{Z}\left(\boldsymbol{x}_{1}\right)-1\right] \cdots\left[\mathcal{Z}\left(\boldsymbol{x}_{k}\right)-1\right] w_{k}\left(\boldsymbol{x}_{1}, \cdots, \boldsymbol{x}_{k}\right) d \boldsymbol{x}_{1} \cdots d \boldsymbol{x}_{k}
$$

Substituting Equation (36) yields

$$
\mathcal{L}_{\mathcal{M}}[\mathcal{X}]=\exp \sum_{k=1}^{\infty} \frac{\bar{n}^{k}}{k !} \int_{\Omega_{\mathrm{b}} \times \cdots \times \Omega_{\mathrm{b}}} \ldots \prod_{j=1}^{k}\left\{\mathcal{L}_{\mathfrak{N}}\left[\int_{\mathbb{R}^{2}} \mathcal{X}(\boldsymbol{x}) h\left(\boldsymbol{x}-\boldsymbol{x}_{j}\right) d \boldsymbol{x}\right]-1\right\} w_{k}\left(\boldsymbol{x}_{1}, \cdots, \boldsymbol{x}_{k}\right) d \boldsymbol{x}_{1} \cdots d \boldsymbol{x}_{k}
$$


Again by letting $\mathcal{X}(\boldsymbol{x})=t$, we have

$$
\begin{aligned}
\mathcal{L}_{f}(t) & \left.=\exp \sum_{k=1}^{\infty} \frac{\bar{n}^{k}}{k !} \int_{\Omega_{\mathrm{b}} \times \cdots \times \Omega_{\mathrm{b}}} \ldots \int_{j=1} \prod_{S_{j}} e^{-t S_{j} h\left(\boldsymbol{x}-\boldsymbol{x}_{j}\right)} \mathfrak{p}\left(S_{j}\right) d S_{j}-1\right] w_{k}\left(\boldsymbol{x}_{1}, \cdots, \boldsymbol{x}_{k}\right) d \boldsymbol{x}_{1} \cdots d \boldsymbol{x}_{k} \\
& =\exp \sum_{k=1}^{\infty} \frac{\bar{n}^{k}}{k !} \int_{\Omega_{\mathrm{b}} \times \cdots \times \Omega_{\mathrm{b}}} \ldots \int_{j=1}^{k} \int_{S_{j}}\left[e^{-t S_{j} h\left(\boldsymbol{x}_{j}\right)}-1\right] \mathfrak{p}\left(S_{j}\right) d S_{j} w_{k}\left(\boldsymbol{x}_{1}, \cdots, \boldsymbol{x}_{k}\right) d \boldsymbol{x}_{1} \cdots d \boldsymbol{x}_{k} .
\end{aligned}
$$

Laplace inversion gives the final general formula for the PDF of intensity fluctuation, $f(I)$.

We obtain cumulants of the PDF of $I$ by the same procedure as Equations (19)-(20):

$$
\begin{aligned}
& \kappa_{1}=\int_{\Omega_{\mathrm{b}}} \int_{S} S h(\boldsymbol{x}) \mathfrak{N}(S) d S d \boldsymbol{x} \\
& \kappa_{2}=\int_{\Omega_{\mathrm{b}}} \int_{S} S^{2} h(\boldsymbol{x})^{2} \mathfrak{N}(S) d S d \boldsymbol{x}+\int_{\Omega_{\mathrm{b}}} \int_{\Omega_{\mathrm{b}}}\left[\prod_{j=1}^{2} \int_{S_{j}} S_{j} h\left(\boldsymbol{x}_{j}\right) \mathfrak{N}\left(S_{j}\right) d S_{j}\right] w_{2} d \boldsymbol{x}_{1} d \boldsymbol{x}_{2},
\end{aligned}
$$

and so on (see Appendix B). It is a natural result that $\kappa_{1}$ is the same as that of unclustered sources. On the other hand, $\kappa_{2}$ involves an additional term with respect to $w_{2}=w_{2}\left(\boldsymbol{x}_{1}, \boldsymbol{x}_{2}\right)$, which causes the fluctuation field overdispersed compared to that of unclustered sources.

\section{RECONSIDERATION OF THE CONFUSION PROBLEM}

The concept of confusion noise and confusion limit is closely related to the fluctuation field $f(I)$. Condon (1974) formulated the confusion limit for a power-law source number counts. His formulation was then extended for general number counts by Franceschini et al. (1989). Takeuchi et al. (2001b) have made some improvement for precise calculation by the method. We should note that the confusion noise affects source counts up to the flux much larger than that of confusion limit (Murdoch et al. 1973). We reconsider the confusion problems in this section.

\subsection{Confusion Noise}

First we review the problems related to the confusion noise. Eddington (1913) proposed an important concept that existence of any statistical noise makes the observed source counts biased upward. Now this well-known phenomenon is called the 'Eddington bias'. He considered the case that the noise is Gaussian. After that, a large amount of efforts has been made to treat and correct the bias by subsequent authors (e.g., Eddington 1940; Bennett 1962; Murdoch et al. 1973). The outline of these ideas is briefly summarized in Murdoch et al. (1973), in comparison with their own method.

Problems with Gaussian noise is beautifully formulated by Murdoch et al. (1973). Their setting assumes that the dispersion of the error does not depend on the source flux. Even in such a simple case, Murdoch et al. (1973) showed that the confusion noise can be very severe for sources with low signal-to-noise ratios. They pointed out that when the confusion noise dominate the error, the total noise cannot be Gaussian, and gave a qualitative discussion on the problem. They claim to use a Monte Carlo approach to evaluate in that case.

However, as we will discuss in the subsequent subsections, we have an expression for the fluctuation field, and we can utilize the information for evaluating the effect of the confusion noise in general cases. Murdoch et al. (1973) properly distinguish between the problems of confusion and blending: the former generally means that the source flux is affected by fainter sources, which cannot be detected by the considered instrument individually, and the latter indicates that the source flux is affected by fainter sources, which is bright enough to be detected as a source if they were located far from the central brightest source. The term 'confusion limit' is related to the former phenomenon, and consequently, confusion limit flux is weaker than the noise flux for bright sources.

How to treat these two effects in a unified manner? Of course they occur in a same way, hence there is no distinction in principle. Consider an ideal situation that there are no detector noise, photon noise, read-out noise, nor any other cosmological diffuse background radiation with structure. Then the noise intensity distribution is the fluctuation PDF itself.

Here we consider a source with true flux $S$. The situation is schematically described in Figure 1 . The source is inevitably affected by other sources in a same beam. The total noise intensity in the beam is $I$. If $I<S$, then the source is observed with flux $S+I$ (see the middle panel of Figure 1). On the other hand, if $I$ is dominated by a bright source $S^{\prime}>S$, then the source is observed as an additive noise of the brighter source with flux $S^{\prime}>S$ (the bottom panel of Figure 1). However, Murdoch et al. (1973) pointed out that there is a possibility that the signal $I$ consists of two or more sources with fluxes fainter than $S$. The probability of such multiple source blending is obtained by the same manner as discussed in $\S 2$. Again we should consider $s_{j}=S_{j} h\left(\boldsymbol{x}-\boldsymbol{x}_{j}\right)$, under the condition

$$
\sum_{j=1}^{k} s_{j}=I,
$$



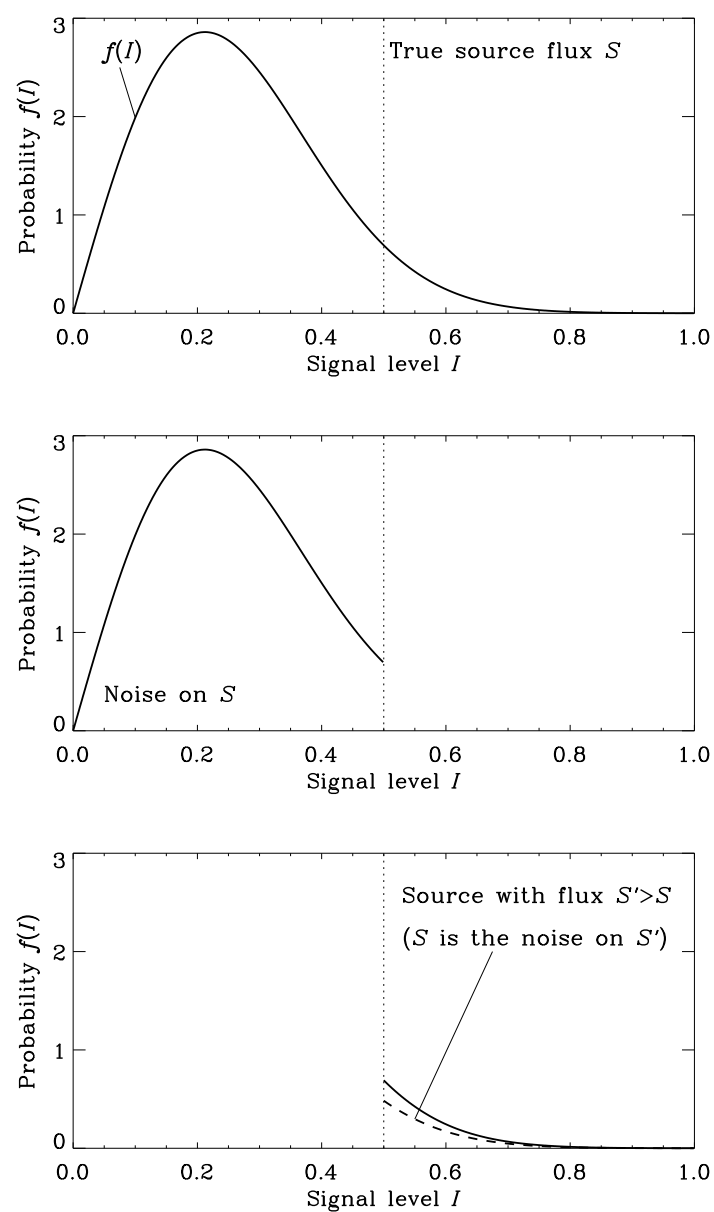

FIG. 1. - A schematic description of the confusion noise problem. Top panel: The source with flux $S$ is embedded in the fluctuation field. Middle panel: The source is observed with flux $S+I$ if the total noise intensity in the beam is $I$. Bottom panel: the source is observed as an additive noise of the brighter source with flux $S^{\prime}>S$ if $I$ is dominated by one or more bright sources $S^{\prime}>S$ (dashed line). On the other hand, if $I$ consists of some sources fainter than $S, I$ should be merely added to $S$ as a noise even if it is larger than $S$.

and the probability of having a noise intensity $I$ produced by $k$ sources is expressed by a convolution [Equation (9)]. For $I$ to be a mere noise, there must not be sources brighter than $S$ in a beam. Hence, we should derive the conditional probability of having $I$ caused by $k$ sources under the condition $S_{j}<S, j=1, \cdots, k$, and then sum up for all $k$ s. It may seem to be a complicated problem, but it can be simplified by Laplace transform. By restricting the range of integration from 0 to $S$ in Equation (15) we have the LT of the conditional PDF as

$$
\mathcal{L}_{g_{k}}(t ; S)=\left[\frac{1}{\Omega_{\mathrm{b}}} \int_{\Omega_{\mathrm{b}}} \int_{0}^{S} e^{-t S^{\prime} h(\boldsymbol{x})} \mathfrak{p}\left(S^{\prime}\right) d S^{\prime} d \boldsymbol{x}\right]^{k} .
$$

Then we have the LT of the desired total conditional PDF, $\tilde{f}(I ; S)$, as

$$
\mathcal{L}_{\tilde{f}}(t ; S)=\exp \bar{n} \int_{\Omega_{\mathrm{b}}}\left[\int_{0}^{S} e^{-t S^{\prime} h(\boldsymbol{x})} \mathfrak{p}\left(S^{\prime}\right) d S^{\prime}-1\right] d \boldsymbol{x}
$$

for unclustered point sources, and

$$
\mathcal{L}_{\tilde{f}}(t ; S)=\exp \sum_{k=1}^{\infty} \frac{\bar{n}^{k}}{k !} \int_{\Omega_{\mathrm{b}} \times \cdots \times \Omega_{\mathrm{b}}} \ldots \prod_{j=1}^{k}\left[\int_{0}^{S} e^{-t S_{j}^{\prime} h\left(\boldsymbol{x}_{j}\right)} \mathfrak{p}\left(S_{j}^{\prime}\right) d S_{j}^{\prime}-1\right] w_{k} d \boldsymbol{x}_{1} \cdots d \boldsymbol{x}_{k}
$$

for clustered sources.

Hence, in order to obtain the confused number counts from the true ones, we should convolve the fluctuation distribution $f(I)$ itself at $I<S$, and $\tilde{f}(I ; S)$ at $I>S$. This consideration naturally explains the fact that the confusion noise flux is stronger than the confusion limit flux. Since we have a variety of background in the image data in a real situation, we fit and subtract from the obtained flux, the noise is not necessarily positive. Thus in practice, the dependence of the confusion noise distribution on flux $S$ causes only a weak variation along $S$ under the existence of other kind of noise, because of the convolution with other noise. 


\subsection{Confusion limit}

We next formulate the relation between the beam size $\theta_{\mathrm{b}}$ and the source confusion limit ${ }^{7}$. The basic procedure is to estimate the limit signal strength as an upper cutoff of the integration in the second-order cumulant formulae in Equation (20) or Equation (48) by iterations. Here we express the second-order cumulant formulae as a function of signal strength $s$ :

$$
\begin{aligned}
\kappa_{2} & =\int_{\Omega_{\mathrm{b}}} \int_{S} S^{2} h(\boldsymbol{x})^{2} \mathfrak{N}(S) d S d \boldsymbol{x} \\
& =\int_{s} s^{2} \int_{\Omega_{\mathrm{b}}} \mathfrak{N}\left[\frac{s}{h(\boldsymbol{x})}\right] \frac{d \boldsymbol{x}}{h(\boldsymbol{x})} d s,
\end{aligned}
$$

for unclustered sources, and

$$
\begin{aligned}
\kappa_{2} & =\int_{\Omega_{\mathrm{b}}} \int_{S} S^{2} h(\boldsymbol{x})^{2} \mathfrak{N}(S) d S d \boldsymbol{x}+\int_{\Omega_{\mathrm{b}}} \int_{\Omega_{\mathrm{b}}} \int_{S_{1}} \int_{S_{2}} S_{1} S_{2} h\left(\boldsymbol{x}_{1}\right) h\left(\boldsymbol{x}_{2}\right) \mathfrak{N}\left(S_{1}\right) \mathfrak{N}\left(S_{2}\right) w_{2}\left(\boldsymbol{x}_{1}, \boldsymbol{x}_{2}\right) d S_{1} d S_{2} d \boldsymbol{x}_{1} d \boldsymbol{x}_{2} \\
& =\int_{s} s^{2} \int_{\Omega_{\mathrm{b}}} \mathfrak{N}\left[\frac{s}{h(\boldsymbol{x})}\right] \frac{d \boldsymbol{x}}{h(\boldsymbol{x})} d s+\int_{s_{1}} \int_{s_{2}} s_{1} s_{2} \int_{\Omega_{\mathrm{b}}} \int_{\Omega_{\mathrm{b}}} \mathfrak{N}\left[\frac{s_{1}}{h\left(\boldsymbol{x}_{1}\right)}\right] \mathfrak{N}\left[\frac{s_{2}}{h\left(\boldsymbol{x}_{2}\right)}\right] w_{2} \frac{d \boldsymbol{x}_{1}}{h\left(\boldsymbol{x}_{1}\right)} \frac{d \boldsymbol{x}_{2}}{h\left(\boldsymbol{x}_{2}\right)} d s_{1} d s_{2} .
\end{aligned}
$$

for clustered sources. We utilize these formulae for deriving confusion limits in the following.

\subsubsection{Confusion Limit for power-law number counts}

We begin our discussion with the case that the number count is described by a power-law, according to Condon $(1974)^{8}$ :

$$
\mathfrak{N}(S)=\alpha S^{-\gamma} \text {. }
$$

For unclustered sources, we obtain the confusion limit flux to a cutoff signal $s_{\mathrm{c}}$ from Equation (52) as

$$
\begin{aligned}
\sigma\left(s_{\mathrm{c}}\right)^{2} & =\int_{0}^{s_{\mathrm{c}}} s^{2} \int_{\Omega_{\mathrm{b}}} \mathfrak{N}\left[\frac{s}{h(\boldsymbol{x})}\right] \frac{d \boldsymbol{x}}{h(\boldsymbol{x})} d s, \\
& =\int_{0}^{s_{\mathrm{c}}} s^{2} \int_{\Omega_{\mathrm{b}}} \alpha h(\theta)^{\gamma-1} s^{-\gamma} \theta d \theta d \phi \\
& \equiv \int_{0}^{s_{\mathrm{c}}} \alpha \Omega_{\mathrm{eff}} s^{2-\gamma} d s=\left(\frac{\alpha \Omega_{\mathrm{eff}}}{3-\gamma}\right) s_{\mathrm{c}}^{3-\gamma},
\end{aligned}
$$

where $\Omega_{\mathrm{eff}}$ is the so-called effective beam size, defined as

$$
\Omega_{\mathrm{eff}} \equiv \int_{\Omega_{\mathrm{b}}} h(\theta)^{\gamma-1} \theta d \theta d \phi .
$$

Taking the square root of Equation (55) and setting $s_{\mathrm{c}}=a \sigma$ as often used, we have

$$
\sigma=\left(\frac{a^{3-\gamma}}{3-\gamma}\right)^{1 /(\gamma-1)}\left(\alpha \Omega_{\mathrm{eff}}\right)^{1 /(\gamma-1)} .
$$

If the beam pattern is described by a Gaussian

$$
h(\theta)=e^{-4 \ln 2\left(\frac{\theta}{\theta_{\mathrm{b}}}\right)^{2}},
$$

where $\theta_{\mathrm{b}}$ is the FWHM of the beam, we get Condon's analytic formula for the $a-\sigma$ confusion limit,

$$
\sigma=\left(\frac{a^{3-\gamma}}{3-\gamma}\right)^{1 /(\gamma-1)}\left[\frac{\pi \theta_{\mathrm{b}}^{2} \alpha}{(4 \ln 2)(\gamma-1)}\right]^{1 /(\gamma-1)} .
$$

It is convenient to relate these expressions and the empirical rule of thumb for the confusion limit. We discuss the relation between the above formulae and the empirical rule of thumb in Appendix C.

Now we turn to the same problem for clustered sources. For clustered sources, the confusion limit can be obtained in similar way by substituting Equation (54) as follows:

$$
\begin{aligned}
\sigma\left(s_{\mathrm{c}}\right)^{2} & =\int_{0}^{s_{\mathrm{c}}} s^{2} \int_{\Omega_{\mathrm{b}}} \mathfrak{N}\left[\frac{s}{h(\boldsymbol{x})}\right] \frac{d \boldsymbol{x}}{h(\boldsymbol{x})} d s+\int_{0}^{s_{\mathrm{c}}} \int_{0}^{s_{\mathrm{c}}} s_{1} s_{2} \int_{\Omega_{\mathrm{b}}} \int_{\Omega_{\mathrm{b}}} \mathfrak{N}\left[\frac{s_{1}}{h\left(\boldsymbol{x}_{1}\right)}\right] \mathfrak{N}\left[\frac{s_{2}}{h\left(\boldsymbol{x}_{2}\right)}\right] w_{2} \frac{d \boldsymbol{x}_{1}}{h\left(\boldsymbol{x}_{1}\right)} \frac{d \boldsymbol{x}_{2}}{h\left(\boldsymbol{x}_{2}\right)} d s_{1} d s_{2} \\
& =\int_{0}^{s_{\mathrm{c}}} s^{2} \int_{\Omega_{\mathrm{b}}} \alpha h(\theta)^{\gamma-1} s^{-\gamma} \theta d \theta d \phi+\int_{0}^{s_{\mathrm{c}}} \int_{0}^{s_{\mathrm{c}}} s_{1} s_{2} \int_{\Omega_{\mathrm{b}}} \int_{\Omega_{\mathrm{b}}} \alpha^{2}\left[h\left(\theta_{1}\right) h\left(\theta_{2}\right)\right]^{\gamma-1}\left(s_{1} s_{2}\right)^{-\gamma} w_{2} \frac{\theta_{1} d \theta_{1} d \phi_{1}}{h\left(\theta_{1}\right)} \frac{\theta_{2} d \theta_{2} d \phi_{2}}{h\left(\theta_{2}\right)} d s_{1} d s_{2} \\
& =\Omega_{\mathrm{eff}}\left(\frac{\alpha}{3-\gamma}\right) s_{\mathrm{c}}^{3-\gamma}+\left\langle\Omega_{\mathrm{eff}}^{2}\right\rangle\left(\frac{\alpha}{2-\gamma}\right)^{2} s_{\mathrm{c}}^{2(2-\gamma)},
\end{aligned}
$$

where we defined the following quantity

$$
\left\langle\Omega_{\mathrm{eff}}^{2}\right\rangle \equiv \int_{\Omega_{\mathrm{b}}} \int_{\Omega_{\mathrm{b}}}\left[h\left(\theta_{1}\right) h\left(\theta_{2}\right)\right]^{\gamma-1} w_{2} \theta_{1} d \theta_{1} d \phi_{1} \theta_{2} d \theta_{2} d \phi_{2}
$$

In general, we cannot solve Equation (60) analytically, hence we should calculate it numerically.

${ }^{7}$ Hereinafter we assume that $h(\boldsymbol{x})=(\theta, \phi)=h(\theta)$ for simplicity. This is not an essential assumption for the subsequent discussions.

${ }^{8}$ Here $\gamma$ is the power-law exponent of differential number counts. This is the same convention with Franceschini (1982). Note that Barcons (1992) uses the same character $\gamma$ as the exponent of cumulative number counts, and consequently the related expressions are apparently different from those in Barcons (1992) in terms of $\gamma$. Hogg (2001) also use the cumulative count slope for his numerical study of the confusion errors. 


\subsubsection{Confusion Limit for general number counts}

Franceschini et al. (1989) presented an iterative formula for the confusion limit of the general number counts. Here we see the result for a Gaussian beam [Equation (58)]:

$$
\begin{aligned}
\sigma^{2}\left(s_{\mathrm{c}}\right) & =\int_{0}^{s_{\mathrm{c}}} s^{2} \int_{\Omega_{\mathrm{b}}} \mathfrak{N}\left[\frac{s}{h(\boldsymbol{x})}\right] \frac{d \boldsymbol{x}}{h(\boldsymbol{x})} d s \\
& =\frac{\pi \theta_{\mathrm{b}}^{2}}{4 \ln 2} \int_{0}^{s_{\mathrm{c}}} s^{2}\left[\int_{1}^{\eta_{\mathrm{b}}} \mathfrak{N}(\eta s) d \eta\right] d s \\
& \equiv \frac{\pi \theta_{\mathrm{b}}^{2}}{4 \ln 2} J\left(s_{\mathrm{c}}\right)
\end{aligned}
$$

where $\eta=e^{4 \ln 2\left(\theta / \theta_{\mathrm{b}}\right)}$, and $\eta_{\mathrm{b}}$ is the upper cutoff that corresponds to the beam area. We again set $s_{\mathrm{c}}=a \sigma$, then we have

$$
\sigma=\sqrt{\frac{\pi J(a \sigma)}{4 \ln 2}} \theta_{\mathrm{b}}
$$

Thus we obtain a general relation between the beam size and the confusion limit as

$$
\theta_{\mathrm{b}}=\sqrt{\frac{4 \ln 2}{\pi J(a \sigma)}} \sigma
$$

Same as the case for power-law number counts, when source clustering takes place, we cannot have a simple formula, but we can still extract some information from the expression. Again by setting the upper cutoff $s_{\mathrm{c}}$ in the integrals in Equation (53), we have

$$
\sigma^{2}\left(s_{\mathrm{c}}\right)=\int_{0}^{s_{\mathrm{c}}} s^{2} \int_{\Omega_{\mathrm{b}}} \mathfrak{N}\left[\frac{s}{h(\boldsymbol{x})}\right] \frac{d \boldsymbol{x}}{h(\boldsymbol{x})} d s+\int_{0}^{s_{\mathrm{c}}} \int_{0}^{s_{\mathrm{c}}} s_{1} s_{2} \int_{\Omega_{\mathrm{b}}} \int_{\Omega_{\mathrm{b}}} \mathfrak{N}\left[\frac{s_{1}}{h\left(\boldsymbol{x}_{1}\right)}\right] \mathfrak{N}\left[\frac{s_{2}}{h\left(\boldsymbol{x}_{2}\right)}\right] w_{2} \frac{d \boldsymbol{x}_{1}}{h\left(\boldsymbol{x}_{1}\right)} \frac{d \boldsymbol{x}_{2}}{h\left(\boldsymbol{x}_{2}\right)} d s_{1} d s_{2} .
$$

If the beam profile is a Gaussian, and some specific functional form is obtained for $w_{k}=w_{k}\left(\boldsymbol{x}_{1}, \cdots, \boldsymbol{x}_{k}\right)$, similar numerical computation can be performed in parallel with Equation (62).

\section{APPLICATION: GALAXY CLUSTERING AND CONFUSION}

\subsection{Hierarchical Ansatz}

As we have discussed above, in order to treat the clustering of the sources, a set of prescribed angular correlation functions to some reasonable order are required. Here we focus on the galaxy clustering as a typical example of the related issues. Of course, we can handle any point sources as far as we have some knowledge of their clustering.

Property of higher-order galaxy clustering still remains a matter of debate (for a comprehensive overview, see Peebles 1980), and various models have been advocated in order to describe the correlation function of galaxies. Generally, a hierarchy of the correlation functions continues to infinite order, hence we should introduce a certain closure relation to cut the sequence.

The hierarchical Ansatz is often employed for the correlation functions, both from phenomenological basis (e.g., Balian \& Schaeffer 1989) and theoretical considerations of BBGKY equations (e.g., Davis \& Peebles 1977; Fry 1984b; Yano \& Gouda 1997). The Ansatz claims that the $k$-point correlation is described by a product of $k-1$ two-point correlation functions. These studies consider the three-dimensional correlations, but we can relate them to two-dimensional ones in a simple way (Peebles 1980; Szapudi \& Szalay 1993). We apply the following hierarchical relations for angular galaxy correlation on the sky (Meiksin, Szapudi, \& Szalay 1992):

$$
w_{k}\left(\boldsymbol{x}_{1}, \cdots, \boldsymbol{x}_{k}\right)=q_{k} \sum_{r_{j}: \text { trees }} w_{2}\left(\boldsymbol{x}_{r_{1}}, \boldsymbol{x}_{r_{2}}\right) \cdots w_{2}\left(\boldsymbol{x}_{r_{k-2}}, \boldsymbol{x}_{r_{k-1}}\right),
$$

where $q_{k}$ is a numerical factor to determine the strength of clustering, and the summation $\sum_{r_{j} \text { :trees }}$ is taken over all tree topologies of graphs between points (Fig. 2). For the details of the expansion, see Fry (1984a,b).

\subsection{Correlation of Infrared Galaxies}

Now we focus on the distribution of infrared galaxies, to have some insight to the confusion in the forthcoming infrared and submillimeter galaxy surveys. Today, many large infrared and submillimeter missions are planned or in progress, and their source confusion limits for galaxies have been calculated for each facility (e.g., Ishii, Takeuchi, \& Sohn 2002; Dole, Lagache, \& Puget 2003). They all assumes the random distribution of galaxies on the sky. In order to obtain the confusion limits more precisely, we must take into account the clustering of infrared galaxies properly.

By using the density moment technique, Meiksin, Szapudi, \& Szalay (1992) estimated the coefficients $q_{3}-q_{8}$ in Equation (66) for IRAS 1.2 Jy sample. We use these values up to $k=4$ to approximate the clustering: $q_{3}=1.25$ and $q_{4}=2.19$. The two-point angular correlation function is

$$
w\left(\boldsymbol{x}_{1}, \boldsymbol{x}_{2}\right)=w\left(\left|\boldsymbol{x}_{1}-\boldsymbol{x}_{2}\right|\right)=w\left(\theta_{12}\right)=\left(\frac{\theta_{12}}{\theta_{0}}\right)^{-\beta},
$$




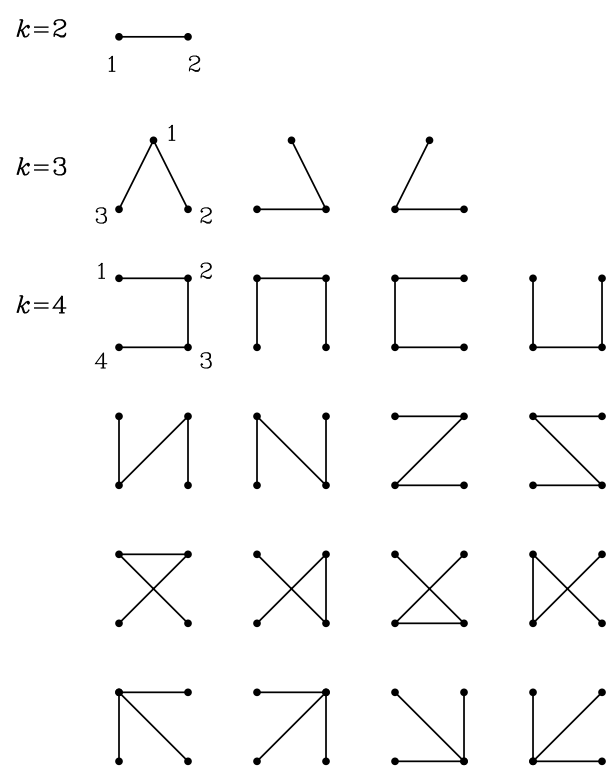

FIG. 2.- The graph representation of the hierarchical expansion of the correlation functions.

and $\beta=0.79, \theta_{0}=0.36$ for the IRAS 1.2 Jy sample. We can obtain the clustering of any deeper surveys via scaling relations of the correlation functions with the characteristic depth of the survey, $d_{*}$ (Peebles 1980):

$$
\begin{aligned}
w_{2}\left(\theta_{12}\right) & =d_{*}^{-1} w_{2}^{0}\left(d_{*} \theta_{12}\right), \\
w_{3}\left(\theta_{12}, \theta_{23}, \theta_{31}\right) & =d_{*}^{-2} w_{3}^{0}\left(d_{*} \theta_{12}, d_{*} \theta_{23}, d_{*} \theta_{31}\right), \\
w_{4}\left(\theta_{12}, \theta_{23}, \theta_{34}, \theta_{41}, \theta_{13}, \theta_{24}\right) & =d_{*}^{-3} w_{4}^{0}\left(d_{*} \theta_{12}, d_{*} \theta_{23}, d_{*} \theta_{34}, d_{*} \theta_{41}, d_{*} \theta_{13}, d_{*} \theta_{24}\right),
\end{aligned}
$$

where superscript 0 represents that it is evaluated at $S=1.2 \mathrm{Jy}$ at $\lambda=60 \mu \mathrm{m}$, i.e., $w_{2}^{0}(\theta)=(\theta / 0.36)^{-0.79}$, and $d_{*}$ is the relative characteristic depth of the survey, defined as $d_{*} \equiv(1.2[\mathrm{Jy}] / S[\mathrm{Jy}])^{1 / 2}$. We observe that the contribution of higher-order clustering rapidly decreases with increasing depth of the survey. This dilution of clustering is more effective for higher-order correlations.

Now we obtain the two-point correlation function $w_{2}(\theta)$ at an arbitrary flux $S$ through Equation (68) as

$$
\begin{aligned}
w_{2}(\theta) & =d_{*}^{-(1+\beta)} w_{2}^{0}(\theta)=d_{*}^{-1.79} w_{2}^{0}(\theta) \\
& =\left(\frac{1.2[\mathrm{Jy}]}{S[\mathrm{Jy}]}\right)^{-1.79 / 2} w_{2}^{0}(\theta) .
\end{aligned}
$$

Consequently, from Equations (69) and (70), we have

$$
\begin{aligned}
w_{3}\left(\theta_{12}, \theta_{23}, \theta_{31}\right) & =d_{*}^{-2(1+\beta)} w_{3}^{0}\left(\theta_{12}, \theta_{23}, \theta_{31}\right) \\
& =d_{*}^{-3.58} w_{3}^{0}\left(\theta_{12}, \theta_{23}, \theta_{31}\right) \\
& =d_{*}^{-3.58} q_{3}\left[w_{2}^{0}\left(\theta_{12}\right)+w_{2}^{0}\left(\theta_{23}\right)+w_{2}^{0}\left(\theta_{31}\right)\right] \\
& =\left(\frac{1.2[\mathrm{Jy}]}{S[\mathrm{Jy}]}\right)^{-3.58 / 2} q_{3}\left[w_{2}^{0}\left(\theta_{12}\right)+w_{2}^{0}\left(\theta_{23}\right)+w_{2}^{0}\left(\theta_{31}\right)\right],
\end{aligned}
$$

and

$$
\begin{aligned}
w_{4}\left(\theta_{12}, \theta_{23}, \theta_{34}, \theta_{41}, \theta_{13}, \theta_{24}\right) & =d_{*}^{-3(1+\beta)} w_{4}^{0}\left(\theta_{12}, \theta_{23}, \theta_{34}, \theta_{41}, \theta_{13}, \theta_{24}\right) \\
& =d_{*}^{-5.37} w_{4}^{0}\left(\theta_{12}, \theta_{23}, \theta_{34}, \theta_{41}, \theta_{13}, \theta_{24}\right) \\
& =d_{*}^{-5.37} q_{4}\left[w_{2}^{0}\left(\theta_{12}\right)+w_{2}^{0}\left(\theta_{23}\right)+w_{2}^{0}\left(\theta_{34}\right)+w_{2}^{0}\left(\theta_{41}\right)+w_{2}^{0}\left(\theta_{13}\right)+w_{2}^{0}\left(\theta_{24}\right)\right] \\
& =\left(\frac{1.2[\mathrm{Jy}]}{S[\mathrm{Jy}]}\right)^{-5.37 / 2} q_{4}\left[w_{2}^{0}\left(\theta_{12}\right)+w_{2}^{0}\left(\theta_{23}\right)+w_{2}^{0}\left(\theta_{34}\right)+w_{2}^{0}\left(\theta_{41}\right)+w_{2}^{0}\left(\theta_{13}\right)+w_{2}^{0}\left(\theta_{24}\right)\right] .
\end{aligned}
$$




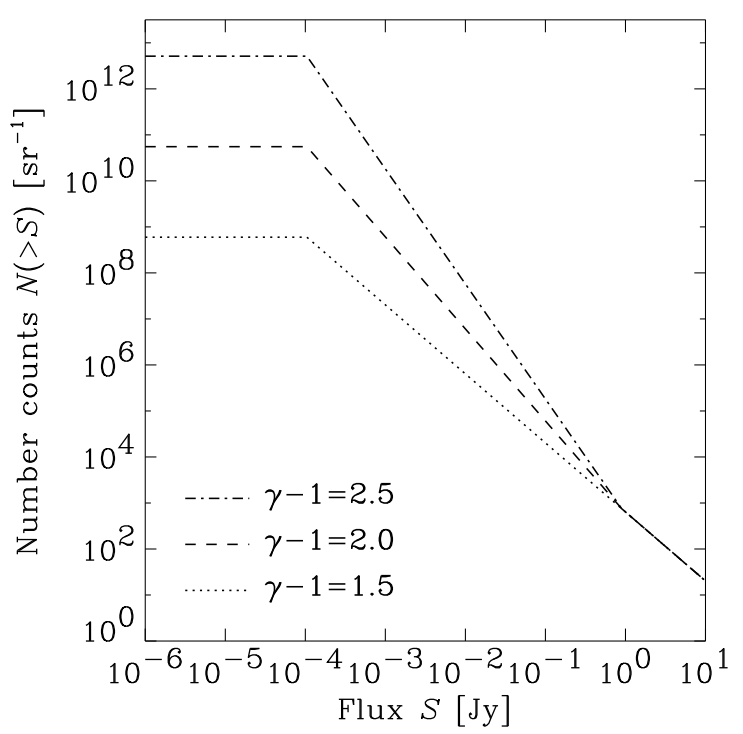

FIG. 3.- The power-law number count model as a model of infrared galaxy counts. These model counts are the integrated ones, hence the power-law indices are represented by $\gamma-1=1.5,2.0$, and 2.5 .

\subsection{Results}

\subsubsection{Power-law number counts}

In order to evaluate how strong the correlation structure affects the confusion-related quantities, we first calculate these statistics based on power-law number count models [Equation (54)],

$$
\mathfrak{N}(S)=\alpha S^{-\gamma}
$$

with the power-law indices $\gamma=2.5,3.0$, and 3.5, i.e., the indices of the integrated counts are $\gamma-1=1.5,2.0$, and 2.5 , respectively. Here, consider a survey at $\lambda=60 \mu \mathrm{m}$. The power-law number counts are normalized so that they have the same counts with the IRAS QMW galaxy survey at a flux $S=0.9$ Jy (Rowan-Robinson et al. 1991). It is observationally known that the bright end of the counts is well approximated by Euclidean, because cosmological and evolutionary effects are both negligible. Actually, IRAS QMW galaxy counts shows a good fit to the Euclidean slope (slope index $\gamma=2.5$ in differential counts), and we can safely fix the slope of the model $\gamma$ to be 2.5 above the flux $S>0.9$ Jy. We estimated the confusion-related statistics for the cases with and without clustering. We assume a telescope with an aperture of $70 \mathrm{~cm}$ with an ideal Airy function as a PSF, and the detection limit of the instrument is $50 \mathrm{mJy}$.

As discussed above, the clustering of galaxies depends on the detected flux: brighter sources have stronger clustering on the sky, and the clustering gradually becomes weaker toward fainter flux. This makes the exact formulation for the statistical characteristic of the two-dimensional galaxy distribution prohibitively difficult (see Barcons 1992), and unfortunately, an exact mathematical theory to treat this problem has not fully established yet. In order to calculate the confusion limit with clustering, we approximate the clustering of the whole sample galaxies evaluated at a 'fiducial' flux, $S_{\text {fid }}$, instead of the flux-dependent clustering in gradual way. Fluctuation consists not only of the detected sources but also of unresolvable sources fainter than detection limit, in principle, toward infinitesimally faint flux. Therefore, the fiducial flux is fainter than the point source detection limit. We assumed that the fiducial flux is proportional to detection limit. Based on this assumption, we calibrated the fiducial flux empirically so as to reproduce the IRAS confusion limit $(\sigma \simeq 20$ mJy: Hacking \& Houck 1987; Lonsdale \& Hacking 1989; Bertin et al. 1997). We found that the relation $S_{\text {fid }}=S_{\text {lim }} / 40$ can be used to evaluate the average clustering strength of the IRAS galaxy sample. Namely, all the galaxies with $S>S_{\text {fid }}$ are approximated to have the same correlation functions $\left[w_{2}(\theta)=d_{\text {fid }}^{-1} w_{2}^{0}\left(d_{\text {fid }} \theta\right)\right.$, etc. $]$ and

TABLE 1

The 5- $\sigma$ CONFUSION LIMITS FOR INFRARED GALAXIES WITH POWER-LAW NUMBER COUNTS.

\begin{tabular}{lcc}
\hline \hline Index & \multicolumn{2}{c}{5 - $\sigma$ confusion limits $[\mathrm{mJ}]$} \\
$\gamma-1$ & Random & Clustering \\
\hline 1.5 & 3.1 & 3.8 \\
2.0 & 22.9 & 120 \\
2.5 & 138 & 9332 \\
\hline
\end{tabular}



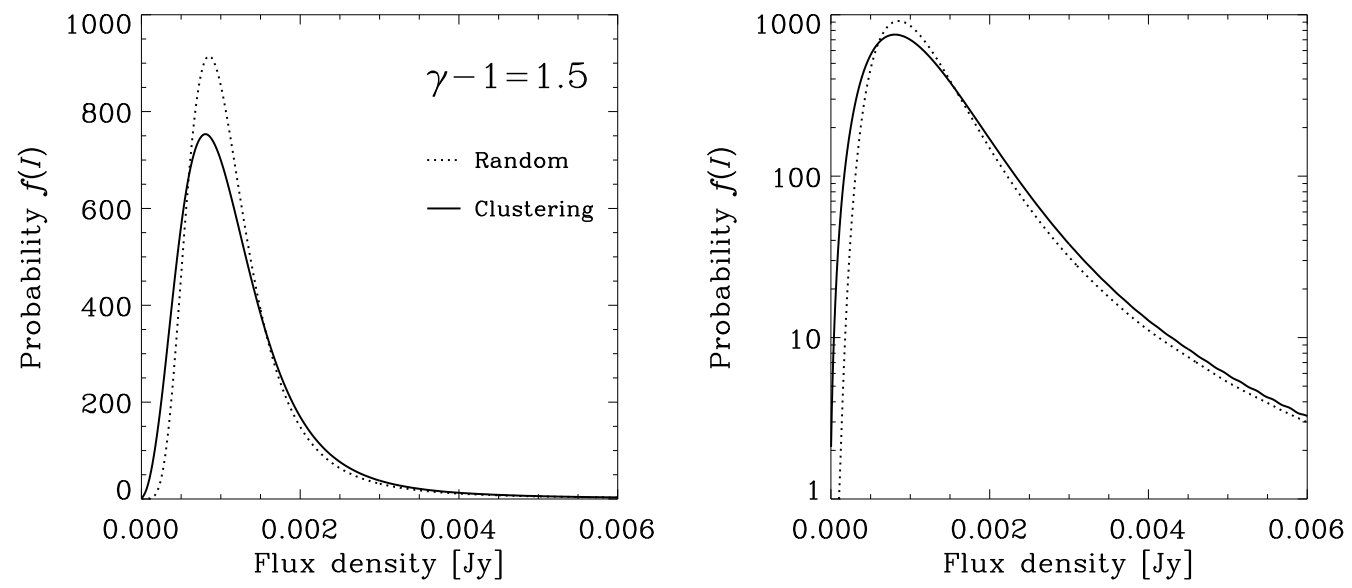

Fig. 4. - The probability density function (PDF) of the fluctuation $f(I)$ for a power-law number count model with a slope index $\gamma=1.5$. We present the PDF in linear scale (Left panel) and in logarithmic scale (Right panel). The dotted lines show the fluctuation distribution for unclustered (randomly distributed) sources, whereas the solid lines represent that for the sources with clustering.
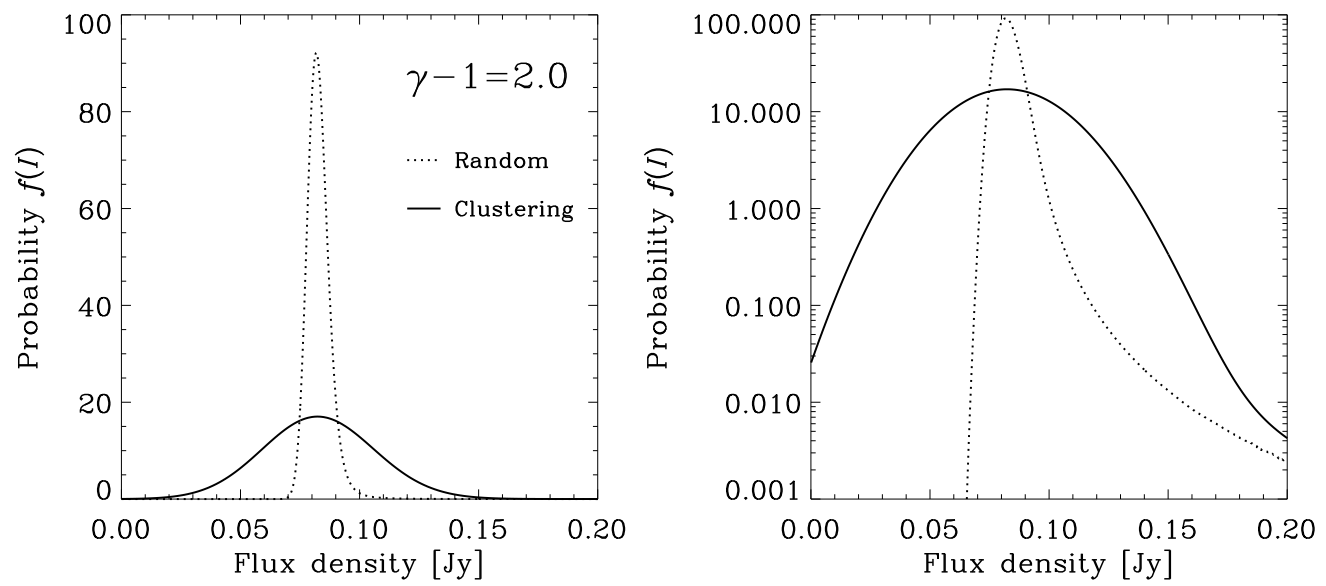

FIG. 5. - The PDF of the fluctuation for a power-law number count model with a slope index $\gamma=2.0$. In contrast to the case of $\gamma=1.5$, the effect of clustering is drastic.

ignore the contribution from the sources with $S<S_{\text {fid }}$. Of course, exact treatment of this part remains to be theoretically improved.

We calculated the confusion limits from the power-law model counts. Without clustering, the confusion limits would be $3.1 \mathrm{mJy}, 22.9 \mathrm{mJy}$, and $138 \mathrm{mJy}$ for the indices $\gamma-1=1.5,2.0$, and 2.5 , respectively. If we take into account the effect of clustering properly, they become $3.8 \mathrm{mJy}, 120 \mathrm{mJy}$, and $9.33 \mathrm{Jy}$, for $\gamma-1=1.5,2.0$, and 2.5, respectively (see Table 1). Thus, the confusion-limit flux get larger, and the effect of clustering strongly depends on the count slope. Especially, if the cumulative count slope $\gamma-1$ exceeds 2.0 , its effect will be catastrophic.

We also obtained the PDF of the fluctuation intensity for infrared galaxies under the same assumptions. Figure 4 shows the PDF with the integral number count slope index $\gamma-1=1.5$. The peak probability decreases by clustering. The clustering also result in a broadening of the PDF (Barcons 1992). It also should noted that the probability of finding a very low intensity at a certain position (closely related to 'the void probability') increases when clustering takes place.

The effect of clustering becomes very strong when the slope index approaches $\gamma-1=2.0$, as presented in Figure 5 . In this case, we have already found that the clustering makes the confusion limit much shallower than that of unclustered case. It means that the rms of the confusion noise is large. Actually, we found that the broadening of the PDF by clustering is very strong, and result in a large variance of the fluctuation PDF (Fig. 5). In summary, the effect of clustering can be very severe if the number counts are described by a single power-law. The above result shows that the relative contribution of clustering to the total fluctuation compared with Poisson component becomes larger for large $\gamma$.

These are consistent with the results reported by Toffolatti et al. (1998) for dusty galaxies at IR wavelengths. As mentioned above, their approach is more model-oriented than the present work, based on the simple model of the spatial two-point correlation function of galaxies. Their central aim was to estimate the power spectrum of the fluctuation in the submillimeter and radio background, and they considered only the two-point correlation, i.e., second-order statistics. Since confusion limit is the second-order quantity, hence both methods can be used, and yield consistent estimates, though 
our method requires only projected information.

Our formulation is fundamentally based on the PDF, and it is the most suitable method of calculating it from the measured projected information (see $\S 2$ ). In order to extend Toffolatti et al.'s method to calculate the PDF, it is necessary to model the bispectrum, trispectrum, and higher-order spectrum or their equivalents (for thorough formulation, see Matsubara 2003). It is a theoretically interesting but challenging problem, and again we see that their method is suitable to theoretical models.

\subsubsection{Realistic number counts for infrared galaxies}

The observed number counts of infrared galaxies cannot be described by a power-law function. Deep galaxy surveys by ISO revealed some striking features of the infrared galaxy counts (see, e.g., Franceschini et al. 2001; Pearson 2001; Takeuchi et al. 2001a; Lagache, Dole \& Puget 2003). Galaxies detected at $90 \mu \mathrm{m}$ or $170 \mu \mathrm{m}$ show a rapid increase in number counts at $S \sim 50-100 \mathrm{mJy}$, with cumulative slope $\gamma-1>2.5$. There have never been any model that succeeded in reproducing the extremely steep number-count slope of ISO sources perfectly, even though many elaborate models have been presented to date.

But such a rapid increase of infrared galaxy cannot be continued toward a fainter flux level, because the integrated infrared flux from galaxies must not exceed the observational constraint of the cosmic infrared background (for the details, see, Takeuchi et al. 2001a; Franceschini et al. 2001; Hauser \& Dwek 2001). This makes a change of the slope of the observed number counts of galaxies.

We show some calculations of the confusion limits for such realistic counts based on an empirical model of Takeuchi et al. (2001a). The parameters are taken from Takeuchi et al. (2002) and Ishii, Takeuchi, \& Sohn (2002). The calculation method is the same for the case of power-law number counts, except that we should use Equation (65) instead of Equation (60).

We obtained the confusion limits of $1.8 \mathrm{mJy}$ for unclustered sources and $2.0 \mathrm{mJy}$ for clustered sources for a model number count at $\lambda=60 \mu \mathrm{m}$. They are $11.4 \mathrm{mJy}$ and $12.3 \mathrm{mJy}$ at $90 \mu \mathrm{m}$, and $105 \mathrm{mJy}$ and $120 \mathrm{mJy}$ at $170 \mu \mathrm{m}$. We summarize these limits in Table 2. Thus in the far-infrared, source clustering does not affect the confusion limit significantly. This is explained as follows: Since the cosmic expansion makes the slope flatter toward fainter fluxes, the count slope becomes sub-Euclidean at the flux level we consider here, in spite of the strong galaxy evolution. The effect of clustering is dominated by the faintest clustered sources, hence the decrease of differential counts makes the effect rather small.

Then, let us consider the PDF of the fluctuation for realistic number counts. We calculated the PDF for wavelengths $\lambda=60,90$, and $170 \mu \mathrm{m}$. Figures 6,7 , and 8 show the PDFs of the fluctuation in these wavelengths. Just as seen in the power-law count models, the peak probability decreases up to $\sim 15 \%$, and the tail of the PDF becomes heavier. We also find an increase of the probability of finding a small flux density. The clustering effect may not seem very severe, because present data generally suffer from more serious noise sources and/or systematics. However, we must keep this effect in mind when we estimate galaxy evolution from the fluctuation analysis of the data that will be obtained by ASTRO-F and SIRTF.

Remind that we assumed the constant clustering strength in this analysis. One may expect a smaller clustering at higher redshift, but biased galaxy formation scenario predicts even stronger clustering for high-redshift dusty galaxies (e.g., Ishii, Takeuchi, \& Enoki 2003). Thus, we should take care of the effect in future infrared data analysis.

\subsubsection{Comments on submillimeter galaxies}

In the submillimeter wavelengths, confusion effects are very serious in single-dish observations (Blain, Ivison, \& Smail 1998; Takeuchi et al. 2001b). Although some large interferometric facilities are under construction, the confusion problem will remain an important issue that should be properly considered for future airborne or space submillimetric observational projects such as BLAST (Balloon-borne Large-Aperture Sub-millimeter Telescope) ${ }^{9}$ and Herschel Space Observatory ${ }^{10}$. As seen above, we need a two-point correlation function of dusty galaxies, whose reliable observational estimate has not been available yet (Scott et al. 2002; Almaini et al. 2003; Borys et al. 2003). For the estimation of the galaxy confusion effect in future submillimeter large surveys (see, e.g., Takeuchi et al. 2001b, and references therein), accurate estimation of the clustering properties is desired. The measured confusion limit from SCUBA survey data was found to be shallower

${ }^{9}$ URL: http://chile1.physics. upenn.edu/blastpublic/index.shtml.

${ }^{10}$ URL: http://astro.estec.esa.nl/First/.

TABLE 2

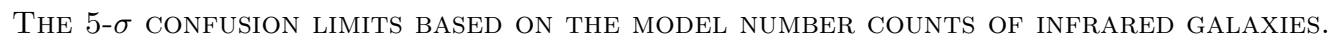

\begin{tabular}{ccc}
\hline \hline $\begin{array}{c}\text { Wavelength } \\
{[\mu \mathrm{m}]}\end{array}$ & \multicolumn{2}{c}{$5-\sigma$ confusion limits $[\mathrm{mJ}]$} \\
Random & Clustering \\
\hline 60 & 1.8 & 2.0 \\
90 & 11.4 & 12.3 \\
170 & 105 & 120 \\
\hline
\end{tabular}



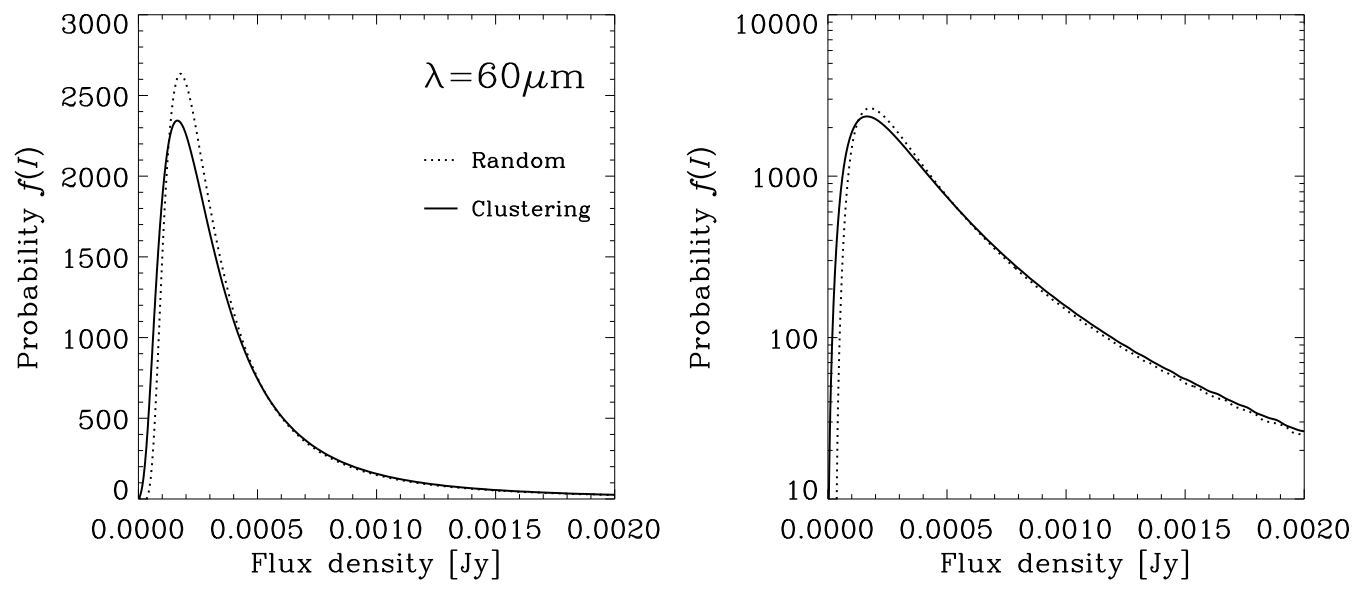

FIG. 6. - The PDF of the fluctuation $f(I)$ for a realistic number count model at $60 \mu \mathrm{m}$ based on Takeuchi et al. (2001a). We present the PDF in linear scale (Left panel) and logarithmic scale (Right panel). The dotted lines show the PDF for unclustered (randomly distributed) sources, whereas the solid lines represent the PDF for clustered sources.
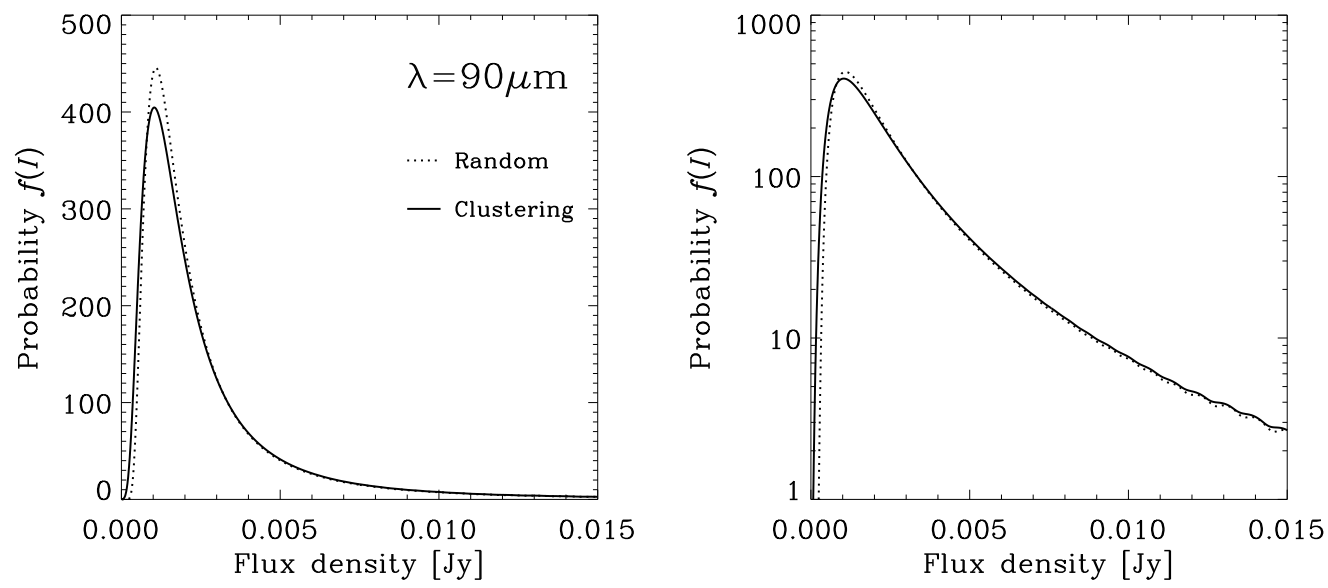

FIG. 7.- Same as Figure 6, except that it is a PDF at $90 \mu \mathrm{m}$.
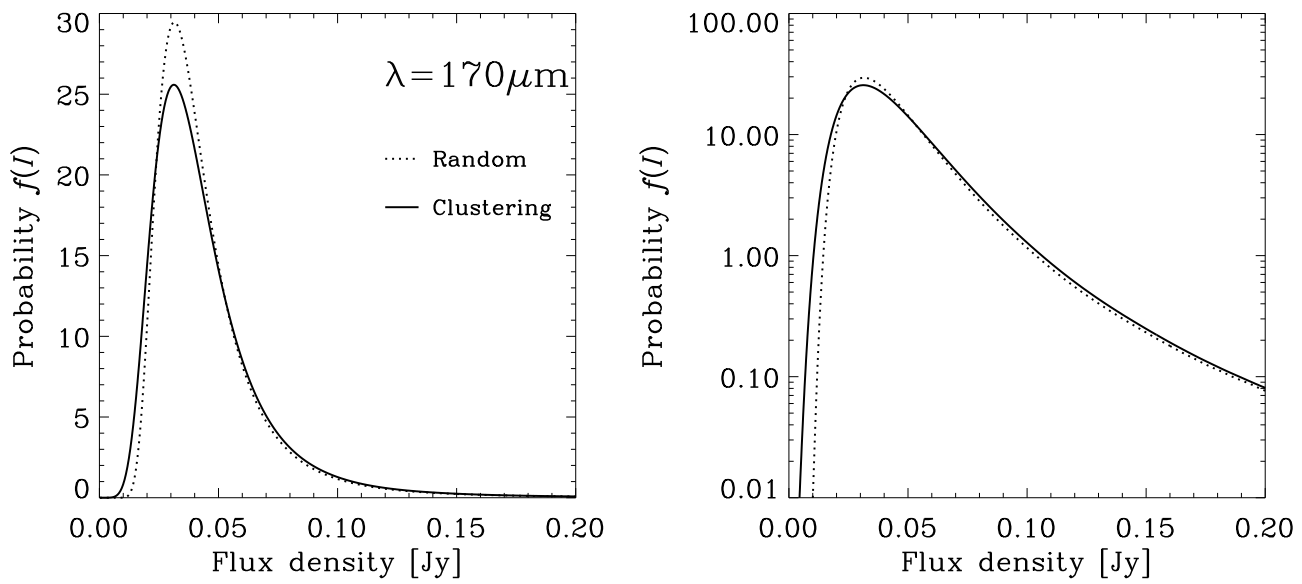

FIG. 8.- Same as Figure 6, except that it is a PDF at $170 \mu \mathrm{m}$.

than that expected from classical formula (D. H. Hughes 2003, private communication), hence the effect of clustering may take place. If we assume that the SCUBA galaxies have the same clustering property with IRAS galaxies, clustering increases the confusion limit from $20 \%$ to $100 \%$, depending on the adopted assumption for the spectral energy distribution of galaxies.

Most of the submillimeter galaxies are dusty vigorous starbursts (e.g., Franceschini et al. 2001; Takeuchi et al. 2001a,b; 
Totani \& Takeuchi 2002), and the dominant constituent of the cosmic submillimeter background radiation. Hence, the statistical structure of the submillimeter background carries crucial information of the spatial distribution of high-redshift starburst galaxies (e.g., Peacock et al. 2000). Our analysis plays an important role as an interface between observed submillimeter data and sophisticated cosmological models (e.g., Magliocchetti et al. 2001; Perrotta et al. 2003).

\section{CONCLUSIONS}

The source confusion is a long-standing problem of the astronomical history, going back to the works of Eddington. Fundamental assumption of the formulation is that the sources are distributed homogeneously on the sky. However, this assumption is not realistic in many applications, e.g., stars in the Galaxy, or galaxies in the Universe. Clustering increases the confusion effect to some extent, but it is not an easy task to formulate the effect in the confusion problem. By using a model for spatial correlation functions, Toffolatti et al. (1998) showed a useful result for the contribution of clustering to the fluctuation of the background at submillimeter and radio wavelengths. Observationally, however, the method to evaluate the confusion only from two-dimensional information of clustering is desirable. In this line, only the work of Barcons (1992) has made attempt to tackle the difficult problem and given solutions to a few simple cases.

In this work, by making extensive use of the methods related to the point field theory, we derived general analytic formulae for the confusion problems with arbitrary distribution and correlation functions. With these formulae, we can obtain the statistical properties of the confusion noise caused by the sources with some prescribed two-dimensional correlation structure.

Based on the general formulae, we first calculated the confusion limits from the power-law galaxy number counts as a test case for the analysis of infrared galaxies. We considered an infrared facility with an aperture of $70 \mathrm{~cm}$ and an ideal Airy PSF. For galaxy clustering, we adopted the hierarchical Ansatz to calculate higher-order correlation functions used in our formulation. Without clustering, the confusion limits would be $3.1 \mathrm{mJy}, 23 \mathrm{mJy}$, and $138 \mathrm{mJy}$ for the indices $\gamma-1=1.5,2.0$, and 2.5 , respectively. If we take into account the effect of clustering properly, they become $3.8 \mathrm{mJy}$, $120 \mathrm{mJy}$, and $9.9 \mathrm{Jy}$, for $\gamma-1=1.5,2.0$, and 2.5 , respectively. We also obtained the probability density function (PDF) of the fluctuation intensity, $f(I)$. We found a broadening of the PDF by clustering, i.e., increase of the variance, corresponding to the increase of the confusion limit flux density. When $\gamma$ approaches to 2.0, the clustering effect becomes very severe for a power-law number counts.

Then we estimated the PDF and confusion limits based on the realistic number count model of Takeuchi et al. (2001a), under the same hypothetical infrared facility. At $60 \mu \mathrm{m}$, we obtained confusion limits of $2.0 \mathrm{mJy}$ for infrared galaxies. At $90 \mu \mathrm{m}$ and $170 \mu \mathrm{m}$, the confusion limits are $12.3 \mathrm{mJy}$ and $120 \mathrm{mJy}$, respectively. These estimates are not much different from the limits calculated without clustering $[1.8 \mathrm{mJy}(60 \mu \mathrm{m}), 11.4 \mathrm{mJy}(90 \mu \mathrm{m})$, and $105 \mathrm{mJy}(170 \mu \mathrm{m})]$. The gradual flattening of the number count slope toward the fainter flux densities, which is observed for infrared galaxies, makes the clustering effect very small, even though the slope is very steep at $S \simeq 10^{-1}-10^{-2} \mathrm{Jy}$. As for the PDF at these wavelength bands, the peak probability also decreases up to $\sim 15 \%$, and the tail of the PDF becomes heavier. The clustering effect may not seem very severe, because present data generally suffer from more serious noise sources and/or systematics. However, we must keep this effect in mind when we estimate galaxy evolution from the fluctuation analysis of the data by $A S T R O-F$ and SIRTF.

We will also apply our method to future submillimeter large surveys. Most of the submillimeter galaxies are dusty vigorous starbursts, and the dominant constituent of the cosmic submillimeter background radiation. Our analysis plays an important role as an interface between observed submillimeter data and sophisticated complex cosmological models.

We sincerely thank the anonymous referee, whose comments and suggestions improved the clarity of this paper very much. We offer our gratitude to Dave Hughes, Motohiro Enoki, Taihei Yano, Haruhiko Ueda, Seiji Ueda, Takuji Tsujimoto, Naoteru Gouda, Yasmin Friedmann, Hiroshi Shibai, and Hiroyuki Hirashita for their helpful and stimulating discussions. We have been supported by the Japan Society of the Promotion of Science.

\section{APPENDIX}

\section{A. FACTORIAL MOMENTS AND CUMULANTS}

We present the definition and some formulae for the important statistical quantities, factorial moments and factorial cumulants here. First we consider a probability generating function (PGF), $G(u)$,

$$
G(u) \equiv \mathrm{E}\left[u^{N}\right],(|u| \leq 1),
$$

where $N$ : a nonnegative integer-valued random variable. Here we define the factorial power. For any integers $n$ and $k$, the factorial powers of $n, n^{[k]}$, as

$$
n^{[k]} \equiv \begin{cases}n(n-1) \cdots(n-k+1) & k=0, \cdots, n \\ 0 & k>n\end{cases}
$$

Factorial moments of $N$ are defined as

$$
m_{[k]} \equiv \mathrm{E}\left[N^{[k]}\right]=\sum_{n=0}^{\infty} \mathrm{p}_{n} n^{[k]}
$$


where

$$
\mathrm{p}_{n} \equiv \operatorname{Prob}\{N=n\} \text {. }
$$

Then the $k$-th factorial moments, $m_{[k]}$ are obtained as the coefficients of the following Taylor expansion,

$$
G(1+v)=1+\sum_{k=1}^{\infty} \frac{m_{[k]} v^{k}}{k !}
$$

Note that we assumed the convergence of the series.

Factorial cumulants, $c_{[k]}$, are similarly calculated by

$$
\ln G(1+v)=1+\sum_{k=1}^{\infty} \frac{c_{[k]} v^{k}}{k !} .
$$

The first few relations are as follows:

$$
\begin{aligned}
c_{[1]} & =m_{[1]}=\mathrm{E}[N], \\
c_{[2]} & =m_{[2]}-m_{[1]}^{2}, \\
c_{[3]} & =m_{[3]}-3 m_{[2]} m_{[1]}+2 m_{[1]}^{3} .
\end{aligned}
$$

For a Poisson distribution, $p_{N}=\bar{n}^{N} e^{-\bar{n}} / N !$,

$$
c_{[1]}=\bar{n}, \quad c_{[k]}=0 \quad(k \geq 2) .
$$

These summary statistics are extensively used in the field of structure formation, especially for counts-in-cells analysis (e.g. Balian \& Schaeffer 1989; Szapudi \& Szalay 1993; Szapudi et al. 1995, 1999). For some more details, see e.g., Vlad et al. (1994) and Kerscher (2001).

\section{B. DERIVATION OF FIRST- AND SECOND-ORDER CUMULANTS FOR GENERAL FIELD}

Here we present how to derive the first- and second-order cumulants for the fluctuation field of clustered sources. We start with the LT [Equation (46)]:

$$
\mathcal{L}_{f}(t)=\exp \sum_{k=1}^{\infty} \frac{\bar{n}^{k}}{k !} \int_{\Omega_{\mathrm{b}} \times \cdots \times \Omega_{\mathrm{b}}} \ldots \prod_{j=1}^{k} \int_{S_{j}}\left[e^{-t S_{j} h\left(\boldsymbol{x}_{j}\right)}-1\right] \mathfrak{p}\left(S_{j}\right) d S_{j} w_{k}\left(\boldsymbol{x}_{1}, \cdots, \boldsymbol{x}_{k}\right) d \boldsymbol{x}_{1} \cdots d \boldsymbol{x}_{k} .
$$

Then, by Equation (18), we observe

$$
\begin{aligned}
\kappa_{1} & =\left.(-1) \frac{d \ln \mathcal{L}_{f}(t)}{d t}\right|_{t=0} \\
& =-\left.\sum_{k=1}^{\infty} \frac{1}{k !} \int_{\Omega_{\mathrm{b}} \times \cdots \times \Omega_{\mathrm{b}}} \ldots \int_{j} \frac{d}{d t}\left\{\prod_{j=1}^{k} \int_{S_{j}}\left[e^{-t S_{j} h\left(\boldsymbol{x}_{j}\right)}-1\right] \mathfrak{N}\left(S_{j}\right) d S_{j}\right\} w_{k} d \boldsymbol{x}_{1} \cdots d \boldsymbol{x}_{k}\right|_{t=0} .
\end{aligned}
$$

Here we define

$$
U\left(\boldsymbol{x}_{j} ; t\right) \equiv \int_{S_{j}}\left[e^{-t S_{j} h\left(\boldsymbol{x}_{j}\right)}-1\right] \mathfrak{N}\left(S_{j}\right) d S_{j}
$$

then Equation (B1) becomes

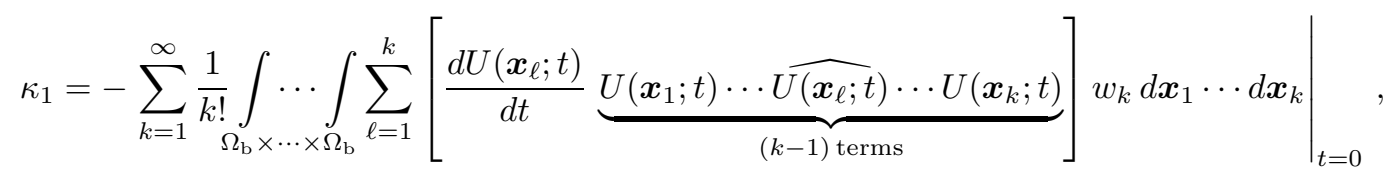

where $\widehat{\cdot}$ means that the term is omitted in the product. We see that

$$
U\left(\boldsymbol{x}_{j} ; 0\right)=\int_{S_{j}}(1-1) \mathfrak{N}\left(S_{j}\right) d S_{j}=0,
$$

therefore, among the terms summed over $k=1, \cdots, \infty$, all those with $k \geq 2$ vanish and only $k=1$ term remains. Thus we obtain

$$
\begin{aligned}
\kappa_{1} & =-\left.\int_{\Omega_{\mathrm{b}}} \frac{d U(\boldsymbol{x} ; t)}{d t} d \boldsymbol{x}\right|_{t=0}=-\left.\int_{\Omega_{\mathrm{b}}} \int_{S}\left[-S h(\boldsymbol{x}) e^{-t S h(\boldsymbol{x})}\right] \mathfrak{N}(S) d S d \boldsymbol{x}\right|_{t=0} \\
& =\int_{\Omega_{\mathrm{b}}} \int_{S} S h(\boldsymbol{x}) \mathfrak{N}(S) d S d \boldsymbol{x} .
\end{aligned}
$$


Next, we consider the second-order cumulant, $\kappa_{2}$ :

$$
\begin{aligned}
& \kappa_{2}=\left.(-1)^{2} \frac{d^{2} \ln \mathcal{L}_{f}(t)}{d t^{2}}\right|_{t=0} \\
& =\left.\frac{d}{d t} \sum_{k=1}^{\infty} \frac{1}{k !} \int_{\Omega_{\mathrm{b}} \times \cdots \times \Omega_{\mathrm{b}}} \ldots \int_{j=1}\left\{\frac{d}{d t} \prod_{j=1}^{k} \int_{S_{j}}\left[e^{-t S_{j} h\left(\boldsymbol{x}_{j}\right)}-1\right] \mathfrak{N}\left(S_{j}\right) d S_{j}\right\} w_{k} d \boldsymbol{x}_{1} \cdots d \boldsymbol{x}_{k}\right|_{t=0} \\
& =\left.\frac{d}{d t} \sum_{k=1}^{\infty} \frac{1}{k !} \int_{\Omega_{\mathrm{b}} \times \cdots \times \Omega_{\mathrm{b}}} \ldots \sum_{\ell=1}^{k}[\frac{d U\left(\boldsymbol{x}_{\ell} ; t\right)}{d t} \underbrace{U\left(\boldsymbol{x}_{1} ; t\right) \cdots \widehat{U\left(\boldsymbol{x}_{\ell} ; t\right)} \cdots U\left(\boldsymbol{x}_{k} ; t\right)}_{(k-1) \text { terms }}] w_{k} d \boldsymbol{x}_{1} \cdots d \boldsymbol{x}_{k}\right|_{t=0} \\
& =\left.\sum_{k=1}^{\infty} \frac{1}{k !} \int_{\Omega_{\mathrm{b}} \times \cdots \times \Omega_{\mathrm{b}}} \ldots \sum_{\ell=1}^{k} \frac{d}{d t}\left[\frac{d U\left(\boldsymbol{x}_{\ell} ; t\right)}{d t} \prod_{j=1 \cdots k}^{j \neq \ell} U\left(\boldsymbol{x}_{j} ; t\right)\right] w_{k} d \boldsymbol{x}_{1} \cdots d \boldsymbol{x}_{k}\right|_{t=0} \\
& =\left.\sum_{k=1}^{\infty} \frac{1}{k !} \int_{\Omega_{\mathrm{b}} \times \cdots \times \Omega_{\mathrm{b}}} \ldots \sum_{\ell=1}\left[\frac{d^{2} U\left(\boldsymbol{x}_{\ell} ; t\right)}{d t^{2}} \prod_{j=1 \cdots k}^{j \neq \ell} U\left(\boldsymbol{x}_{j} ; t\right)+\sum_{\ell, m=1 \cdots k}^{\ell \neq m} \frac{d U\left(\boldsymbol{x}_{\ell} ; t\right)}{d t} \frac{d U\left(\boldsymbol{x}_{m} ; t\right)}{d t} \prod_{j=1 \cdots k}^{j \neq \ell, m} U\left(\boldsymbol{x}_{j} ; t\right)\right] w_{k} d \boldsymbol{x}_{1} \cdots d \boldsymbol{x}_{k}\right|_{t=0} .
\end{aligned}
$$

Among the first term in the square brackets [.] of Equation (B6), all the summands with $k \geq 2$ vanish by substituting $t=0$. On the other hand, the second term in [.] exists only if $k \geq 2$. Further, all the summands with $k \geq 3$ vanish by setting $t=0$. Considering all the above items together, we obtain

$$
\begin{aligned}
\kappa_{2}= & \left.\int_{\Omega_{\mathrm{b}}} \frac{d^{2} U(\boldsymbol{x} ; t)}{d t^{2}} d \boldsymbol{x}\right|_{t=0}+\left.\frac{1}{2 !} \int_{\Omega_{\mathrm{b}}} \int_{\Omega_{\mathrm{b}}}\left[\frac{d U\left(\boldsymbol{x}_{1} ; t\right)}{d t} \frac{d U\left(\boldsymbol{x}_{2} ; t\right)}{d t}+\frac{d U\left(\boldsymbol{x}_{2} ; t\right)}{d t} \frac{d U\left(\boldsymbol{x}_{1} ; t\right)}{d t}\right] w_{2}\left(\boldsymbol{x}_{1}, \boldsymbol{x}_{2}\right) d \boldsymbol{x}_{1} d \boldsymbol{x}_{2}\right|_{t=0} \\
= & \left.\int_{\Omega_{\mathrm{b}}} \frac{d^{2} U(\boldsymbol{x} ; t)}{d t^{2}} d \boldsymbol{x}\right|_{t=0}+\left.\frac{2}{2 !} \int_{\Omega_{\mathrm{b}}} \int_{\Omega_{\mathrm{b}}} \frac{d U\left(\boldsymbol{x}_{1} ; t\right)}{d t} \frac{d U\left(\boldsymbol{x}_{2} ; t\right)}{d t} w_{2}\left(\boldsymbol{x}_{1}, \boldsymbol{x}_{2}\right) d \boldsymbol{x}_{1} d \boldsymbol{x}_{2}\right|_{t=0} \\
= & \left.\int_{\Omega_{\mathrm{b}}} \int_{S} S^{2} h(\boldsymbol{x})^{2} e^{-t S h(\boldsymbol{x})} \mathfrak{N}(S) d S d \boldsymbol{x}\right|_{t=0} \\
& +\left.\int_{\Omega_{\mathrm{b}}} \int_{\Omega_{\mathrm{b}}}\left[\int_{S_{1}} S_{1} h\left(\boldsymbol{x}_{1}\right) e^{-t S_{1} h\left(\boldsymbol{x}_{1}\right)} \mathfrak{N}\left(S_{1}\right) d S_{1} \int_{S_{2}} S_{2} h\left(\boldsymbol{x}_{2}\right) e^{-t S_{2} h\left(\boldsymbol{x}_{2}\right)} \mathfrak{N}\left(S_{2}\right) d S_{2}\right] w_{2}\left(\boldsymbol{x}_{1}, \boldsymbol{x}_{2}\right) d \boldsymbol{x}_{1} d \boldsymbol{x}_{2}\right|_{t=0} \\
= & \int_{\Omega_{\mathrm{b}}} \int_{S} S^{2} h(\boldsymbol{x})^{2} \mathfrak{N}(S) d S d \boldsymbol{x}+\int_{\Omega_{\mathrm{b}}} \int_{\Omega_{\mathrm{b}}}\left[\int_{S_{1}} S_{1} h\left(\boldsymbol{x}_{1}\right) \mathfrak{N}\left(S_{1}\right) d S_{1} \int_{S_{2}} S_{2} h\left(\boldsymbol{x}_{2}\right) \mathfrak{N}\left(S_{2}\right) d S_{2}\right] w_{2}\left(\boldsymbol{x}_{1}, \boldsymbol{x}_{2}\right) d \boldsymbol{x}_{1} d \boldsymbol{x}_{2} .
\end{aligned}
$$

\section{THE EMPIRICAL RULE OF THUMB FOR THE CONFUSION LIMIT}

The ' $1 / 30$ sources per beam' rule is well known to observational astronomers as an empirical convention to avoid confusion effects. This problem was first theoretically addressed by Franceschini (1982). Here we extend his discussion and present a numerical result for the case of Airy beam. According to the notation of Hogg (2001), we use the notation $(s / b)$ as the number of sources per beam:

$$
(s / b)(S) \equiv \Omega_{\mathrm{b}} \int_{S}^{\infty} \mathfrak{N}\left(S^{\prime}\right) d S^{\prime} .
$$

If we would like to detect $a-\sigma$ sources safely, where $\sigma$ is the confusion limit [Equation (57)], the rule of thumb is expressed as

$$
(s / b)_{\mathrm{C}}=\Omega_{\mathrm{b}} \int_{a \sigma}^{\infty} \mathfrak{N}(S) d S,
$$

where the subscript $\mathrm{C}$ represents that $(s / b)$ at the confusion limit. For power-law source counts, we can obtain a useful analytic expression for $(s / b)_{\mathrm{C}}$ as follows:

$$
\begin{aligned}
(s / b)_{\mathrm{C}} & =\Omega_{\mathrm{b}} \int_{a \sigma}^{\infty} \alpha S^{-\gamma} d S \\
& =\frac{\alpha \Omega_{\mathrm{b}}}{\gamma-1}(a \sigma)^{1-\gamma} \\
& =\frac{\alpha \Omega_{\mathrm{b}}}{\gamma-1} a^{1-\gamma}\left(\frac{a^{3-\gamma}}{3-\gamma}\right)^{(1-\gamma) /(\gamma-1)}\left(\alpha \Omega_{\mathrm{eff}}\right)^{(1-\gamma) /(\gamma-1)} \\
& =\frac{\alpha \Omega_{\mathrm{b}}}{\gamma-1} a^{1-\gamma}\left(\frac{a^{3-\gamma}}{3-\gamma}\right)^{-1}\left(\alpha \Omega_{\mathrm{eff}}\right)^{-1} \\
& =\frac{\Omega_{\mathrm{b}}}{\Omega_{\mathrm{eff}}}\left(\frac{3-\gamma}{\gamma-1}\right) a^{-2} .
\end{aligned}
$$




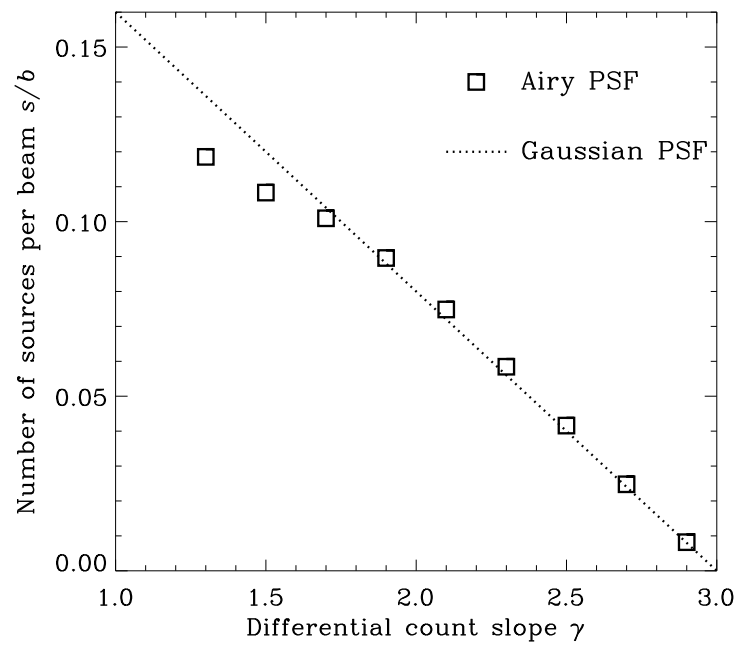

FIG. 9.- The rule of thumb for the confusion limit of $5-\sigma$ sources. Dotted line represents the number of sources per beam $(s / b)_{\mathrm{C}}$ for the confusion limit of a Gaussian beam, as a function of the slope $\gamma$ of the differential source counts. Open squares are $(s / b)_{\mathrm{C}}$ for an Airy beam. These estimates are calculated for unclustered sources.

This corresponds to the result obtained for the general case by Franceschini (1982) in his Equation (14). For a Gaussian beam, Equation (C3) becomes simpler. If we set $\Omega_{\mathrm{b}}=\pi\left(\epsilon \sigma_{\mathrm{G}}\right)^{2}$, where $\sigma_{\mathrm{G}}=\theta_{\mathrm{b}} / 2 \sqrt{2 \ln 2}$ denotes the standard deviation of the Gaussian beam profile, we have

$$
\frac{\Omega_{\mathrm{b}}}{\Omega_{\mathrm{eff}}}=\frac{\epsilon^{2}(\gamma-1)}{2}
$$

Here $\epsilon$ is a numerical factor that depends on the details of the source detection algorithm and beam profile. We regard $\epsilon=2$ as a reasonable choice. Hogg (2001) defined $\Omega_{\mathrm{b}}$ to be $\pi{\sigma_{\mathrm{G}}}^{2}$, which is different from our convention by a factor of $\epsilon$. Then, we observe the following formula

$$
(s / b)_{\mathrm{C}}=\frac{\epsilon^{2}(3-\gamma)}{2 a^{2}} .
$$

This formula clearly shows the dependence of the confusion rule of thumb, $(s / b)_{\mathrm{C}}$ : the steeper the slope is, the more severe the confusion becomes. This trend has already known to observational astronomers empirically, and confirmed by the comprehensive work of Hogg (2001). We tabulate the rule of thumb when we take $a=5$ and $\epsilon=2$ in Table 3 .

Generally, an ideal beam pattern of an optical instrument is often described by an Airy function. We calculated the rule of thumb for an Airy beam by numerical integration. This is shown in Figure 9. The numbers of sources per beam for Gaussian and Airy beam show an excellent agreement at $\gamma>1.7$. However, we find a slight deviation from Gaussian value at $1.3 \lesssim \gamma \lesssim 1.7$ for $(s / b)_{\mathrm{C}}$ of an Airy beam. This may stem from the structure of the Airy function.

\section{GLOSSARY OF MATHEMATICAL SYMBOLS}

We tabulate the mathematical symbols used in the main text in Table 4, for the readers' convenience. We tabulate the equations or sections by which these symbols are defined or explained.

TABLE 3

The Rule of Thumb For the CONfusion limit of 5- $\sigma$ sources, When the Beam is Gaussian.

\begin{tabular}{cc}
\hline \hline $\begin{array}{c}\text { Index } \\
\gamma\end{array}$ & $\begin{array}{c}\text { Sources per beam } \\
(s / b)_{\mathrm{C}}\end{array}$ \\
\hline 0.5 & 0.20 \\
1.0 & 0.16 \\
1.5 & 0.12 \\
2.0 & 0.08 \\
2.5 & 0.04 \\
2.9 & 0.008 \\
\hline
\end{tabular}


TABLE 4

Glossary of mathematical Symbols.

\begin{tabular}{|c|c|c|}
\hline Symbol & Definition & Equation \\
\hline Prob $\{$ Event $\}$ & Probability that a certain event occurs & (5) \\
\hline $\mathrm{E}[X]$ & Expectation value of a random variable $X$ & (6) \\
\hline $\mathbb{I}_{A}$ & Indicator function of a set $A$ & (34) \\
\hline $\mathbb{R}^{2}$ & Real plane & $\S 2.1 .2$ \\
\hline$\Omega_{\mathrm{b}}$ & Beam area of an instrument on the sky & $(33)$ \\
\hline$\Omega_{\text {eff }}$ & Effective beam size, i.e., beam area weighted by $h(\theta)^{\gamma-1}$ & $(55)$ \\
\hline$\left\langle\Omega_{\mathrm{eff}}^{2}\right\rangle$ & Squared beam area weighted by $h\left(\theta_{1}\right)^{\gamma-1}, h\left(\theta_{2}\right)^{\gamma-1}$, and $w_{2}\left(\theta_{12}\right)$ & $(60)$ \\
\hline$\alpha$ & Proportional constant for a power-law source number counts & (54) \\
\hline$\beta$ & Slope index of the power-law angular correlation function of galaxies & (67) \\
\hline$\gamma$ & Exponent of a power-law source number counts & $(54)$ \\
\hline$\theta, \phi, \theta_{1}, \theta_{2}, \cdots$ & Angular coordinate or separation on the sky & $(55)$ \\
\hline$\theta_{i j}$ & Angular separation between objects $i$ and $j$ & (67) \\
\hline$\theta_{\mathrm{b}}$ & FWHM of the beam pattern & (58) \\
\hline$\theta_{0}$ & Correlation scale length of angular correlation & 67 \\
\hline$\kappa_{k}$ & $k$-th order cumulant of a random variable & (18) \\
\hline$\mu_{k}$ & $k$-th order moment of a random variable & (17) \\
\hline$\sigma$ & Confusion limit flux to a cutoff of $a-\sigma$ level & (57) \\
\hline$\sigma\left(s_{\mathrm{C}}\right)$ & Confusion limit flux to a cutoff signal $s_{\mathrm{C}}$ & $(55)$ \\
\hline$\sigma_{\mathrm{G}}$ & Standard deviation of a Gaussian beam profile & Appendix $\mathrm{C}$ \\
\hline$G[\mathcal{Z}]$ & Probability generating functional (PGFL) of a point field & $(32)$ \\
\hline$I(\boldsymbol{x})$ & Intensity of a signal at a position $\boldsymbol{x}$ & $(2)$ \\
\hline$I_{N}(\boldsymbol{x})$ & Intensity of a signal at a position $\boldsymbol{x}$, consisting of exactly $N$ sources & (3) \\
\hline $\mathcal{L}_{f}(t)$ & Laplace transform $(\mathrm{LT})$ of $f(t)$ & (6) \\
\hline $\mathcal{L}_{\tilde{f}}(t ; S)$ & LT of $\tilde{f}$ under the condition that any $S_{i}<S$ for any $i=0,1,2, \cdots$ & $(50)$ \\
\hline $\mathcal{L}_{g}^{J}(t)$ & LT of $g(t)$ & $(13)$ \\
\hline $\mathcal{L}_{g_{N}}(t)$ & LT of $g_{N}(t)$ & $(7)$ \\
\hline $\mathcal{L}_{g_{k}}(t ; S)$ & LT of $g_{k}$ under the condition that any $S_{i}<S$ for any $i=1, \cdots, k$ & (49) \\
\hline $\mathcal{L}^{-1}[u]$ & Inverse Laplace transform of a certain function $u(t)$ & (16) \\
\hline $\mathcal{L}_{\mathcal{M}}[\mathcal{X}]$ & Laplace functional of a test function $\mathcal{X}$ with respect to a measure $\mathcal{M}$ & (24) \\
\hline $\mathcal{L}_{\mathfrak{N}}[\mathcal{U}]$ & Laplace-Stieltjes functional of $\mathcal{U}$ with respect to $\mathfrak{p}(S)$ & (26) \\
\hline $\mathcal{M}(A)$ & Random measure a random field with respect to an area $A$ & (23) \\
\hline$N$ & Measured number of sources in a beam & $(3)-(5)$ \\
\hline $\mathcal{N}(A)$ & Counting measure of a point field in an area $A$ & $(22)$ \\
\hline $\mathfrak{N}(S)$ & Differential source number counts with a flux density $S$ & $\S 2.1 .1$ \\
\hline$S$ & Detected flux density of a source & $\S 2.1 .1$ \\
\hline$S_{n}$ & Detected flux density of a source with label $n$ & $\S 2.1 .1$ \\
\hline$S_{\lim }$ & Detection limit flux for point sources & $\S 4.3 .1$ \\
\hline$S_{\text {fid }}$ & Fiducial flux to approximate the average correlation of a sample & $\S 4.3 .1$ \\
\hline $\mathcal{X}$ & Test function, with which a value is fixed for a functional & $(24)$ \\
\hline $\mathcal{Y}$ & Test function of a functional & (29) \\
\hline $\mathcal{Z}\left(\boldsymbol{x}_{n}\right)$ & Laplace-Stieltjes functional of $\int_{\mathbb{R}^{2}} \mathcal{X}(\boldsymbol{x}) h\left(\boldsymbol{x}-\boldsymbol{x}_{n}\right) d \boldsymbol{x}$ & $(26),(28)$ \\
\hline $\mathcal{Z}^{*}\left(\boldsymbol{x}_{n}\right)$ & Test function $\mathcal{Z}\left(\boldsymbol{x}_{n}\right)$ but locally restricted within a beam & $(33)$ \\
\hline$a$ & Flux density level measured by standard deviation of the noise & $\S 3.2 .1$ \\
\hline$c_{[k]}$ & $k$-th factorial cumulant & $(29),(\mathrm{A} 6)$ \\
\hline$d_{*}$ & Relative characteristic depth of a survey with respect to $S=1.2 \mathrm{Jy}$ & $(68)-(70)$ \\
\hline$f(I)$ & Probability density function (PDF) of the signal intensity of the image & $(5)$ \\
\hline$g(I)$ & PDF of $I_{1}$, i.e., signal produced by a single source & $(10),(11)$ \\
\hline$g_{N}(I)$ & PDF of $I_{N}$, i.e., signal produced by exactly $N$ sources & $(5),(9)$ \\
\hline$h(\boldsymbol{x})$ & Beam pattern of the instrument & $\S 2.1 .1$ \\
\hline$m_{[k]}$ & $k$-th factorial moment & (A5) \\
\hline $\bar{n}$ & Mean of a source number density on the sky, $n_{\ell}(\ell=1,2, \ldots)$ & $\S 2.1 .1$ \\
\hline$n^{[k]}$ & $k$-th factorial power of a certain integer $n$ & (A2) \\
\hline$p_{N}$ & Probability of obtaining a number $N$ for a Poisson random variable & $(4)$ \\
\hline $\mathfrak{p}(S)$ & Probability of finding a source with a flux $S$ & $\S 2.1 .1$ \\
\hline$q_{k}$ & Coefficient of the hierarchical model for the $k$-point correlation function & (66) \\
\hline$s(\boldsymbol{x})$ & Intensity of the signal at position $\boldsymbol{x}, s(\boldsymbol{x}) \equiv \operatorname{Sh}(\boldsymbol{x})$ & $\S 2.1 .1$ \\
\hline$s_{\mathrm{C}}$ & Cutoff flux level used for calculating confusion limit & (55) \\
\hline$s_{n}(\boldsymbol{x})$ & Intensity of the signal at $\boldsymbol{x}$ produced by a single source at $\boldsymbol{x}_{n}$ & $\S 2.1 .1$ \\
\hline$(s / b)_{\mathrm{C}}$ & Number of sources per beam at the confusion limit & (C3) \\
\hline$(s / b)(S)$ & Number of sources per beam to a flux limit $S$ & (C1) \\
\hline$w_{k}\left(\boldsymbol{x}_{1}, \cdots, \boldsymbol{x}_{k}\right)$ & Angular $k$-point correlation function & $(29),(67)$ \\
\hline$w_{k}^{0}\left(\boldsymbol{x}_{1}, \cdots, \boldsymbol{x}_{k}\right)$ & Angular $k$-point correlation function evaluated at $S=1.2 \mathrm{Jy}$ & $(68)-(70)$ \\
\hline & Position where the intensity of the field is measured & $\S 2.1 .1$ \\
\hline $\boldsymbol{x}_{n}$ & Position of a source with label $n$ & $\S 2.1 .1$ \\
\hline
\end{tabular}




\section{REFERENCES}

Almaini, O., et al. 2003, MNRAS, 338, 303

Barcons, X., \& Fabian, A. C. 1988, MNRAS, 230, 189

Barcons, X., \& Fabian, A. C. 1990, MNRAS, 243, 366

Barcons, X. 1992, ApJ, 396, 460

Barcons, X., Branduardi-Raymont, G., Warwick, R. S., Fabian, A. C., Mason, K. O., McHardy, I., \& Rowan-Robinson, M. 1994, MNRAS, 268, 833

Barcons, X., Fabian, A. C., \& Carrera, F. J. 1998, MNRAS, 293, 60

Balian, R., \& Schaeffer, R. 1989, A\&A, 220, 1

Bennett, A. S. 1962, MNRAS, 125, 75

Bertin, E., Dennefeld, M., \& Moshir, M. 1997, A\&A, 323, 685

Blain, A. W., Ivison, R. J., \& Smail, I. 1998, MNRAS, 296, L29

Borys, C., Chapman, S., Halpern, M., \& Scott, D. 2003, MNRAS, 344,385

Burigana, C., \& Popa, L. 1998, A\&A, 334, 420

Campbell, N. R. 1909, Proc. Cambridge Phil. Soc., 15, 117

Chandrasekhar, S., \& Münch, G. 1950, ApJ, 112, 380

Condon, J. J. 1974, ApJ, 188, 279

Condon, J. J., \& Dressel, L. L. 1978, ApJ, 222, 745

Crawford D. F., Jauncey D. L., \& Murdoch H. S. 1970, ApJ, 162, 405

Cressie, N. A. C. 1993, Statistics for Spatial Data, John Wiley \& Sons, Inc., New York

Daley, D. J., \& Vere-Jones, D. 2003, An Introduction to the Theory of Point Processes, Volume I: Elementary Theory and Methods, 2nd ed. (New York: Springer)

Davis, M., \& Peebles, P. J. E. 1977, ApJS, 34, 425

Dole, H., Lagache, G., \& Puget, J.-L. 2003, ApJ, 585, 617

Eales, S., Lilly, S., Webb, T., Dunne, L., Gear, W., Clements, D., \& Yun, M. 2000, AJ, 120, 2244

Eddington, A. S. 1913, MNRAS, 73, 359

Eddington, A. S. 1940, MNRAS, 100, 354

Franceschini, A. 1982, Ap\&SS, 86, 3

Franceschini, A., Toffolatti, L., Danese, L., \& de Zotti, G. 1989, ApJ, 344, 35

Franceschini, A., Toffolatti, L., Mazzei, P., Danese, L., \& de Zotti, G. 1991, A\&AS 89, 285

Franceschini, A., Aussel, H., Cesarsky, C. J., Elbaz, D., \& Fadda, D. 2001, A\&A, 378, 1

Friedmann, Y., \& Bouchet, F. 2003, MNRAS, in press (astro-ph/0310785)

Frisch, U. 1995, Turbulence (Cambridge: Cambridge Univ. Press)

Fry, J. N. 1984a, ApJ, 277, L5

Fry, J. N. 1984b, ApJ, 279, 499

Guiderdoni, B., Hivon, E., Bouchet, F. R., \& Maffei, B. 1998, MNRAS, 295, 877

Hacking, P., \& Houck, J. R. 1987, ApJS, 63, 311

Hauser, M. G. \& Dwek, E. 2001, ARA\&A, 39, 249

Hewish, A. 1961, MNRAS, 123, 167

Hirashita, H., Takeuchi, T. T., Shibai, H., \& Ohta, K. 1999, PASJ, 51,81

Hogg, D. W. 2001, AJ, 121, 1207

Hogg, D. W., \& Turner, E. L. 1998, PASP, 110, 727

Ishii, T. T., Takeuchi, T. T., \& Sohn, J.-J. 2002, in Infrared and Submillimeter Space Astronomy, ed. M. Giard, J. P. Bernard, A. Klotz, \& I. Ristrorcelli (Les Ulis: EDP Sciences), 369

Ishii, T. T., Takeuchi, T. T., \& Enoki, M. 2003, in Multiwavelength Cosmology, ed. M. Plionis \& I. Georgantopoulos (Dordrecht: Kluwer), in press
Kerscher, M. 2001, Phys. Rev. E, 64, 56109

Lagache, G., \& Puget, J.-L. 2000, A\&A, 355, 17

Lagache, G., Dole, H., \& Puget, J.-L. 2003, MNRAS, 338, 555

Lonsdale, C. J., \& Hacking, P. B. 1989, ApJ, 339, 712

Ma, S.-K. 1985, Statistical Mechanics (Philadelphia: World Scientific)

Magliocchetti, M., Moscardini, L., Panuzzo, P., Granato, G. L., De Zotti, G., \& Danese, L. 2001, MNRAS, 325, 1553

Matsubara, T. 1995, ApJS, 101, 1

Matsubara, T. 2003, ApJ, 584, 1

Matsuhara, H., et al. 2000, A\&A, 361, 407

Meiksin, A., Szapudi, I., \& Szalay, A. 1992, ApJ, 394, 87

Miville-Deschênes, M.-A., Lagache, G., \& Puget, J.-L. 2002, A\&A, 393,749

Murdoch H. S., Crawford D. F., \& Jauncey D. L. 1973, ApJ, 183, 1

Oliver, S., et al. 1997, MNRAS, 289, 471

Peacock, J. A., et al. 2000, MNRAS, 318, 535

Pearson, C. P. 2001, MNRAS, 325, 1511

Peebles, P. J. E. 1980, Large Scale Structure of the Universe (Princeton: Princeton Univ. Press)

Perrotta, F., Magliocchetti, M., Baccigalupi, C., Bartelmann, M., De Zotti, G., Granato, G. L., Silva, L., \& Danese, L. 2003, MNRAS, 338, 623

Refsdal, S. 1969, ApJ, 155, 373

Rice, S. O. 1944, Bell System Tech. J., 23, 282 [reprinted in Selected Papers on Noise and Stochastic Processes, 2003, ed., N. Wax, (New York: Dover)]

Rowan-Robinson, M., Saunders, W., Lawrence, A., \& Leech, K. 1991, MNRAS, 253, 485

Scheuer, P. A. G. 1957, Proc. Cambridge Phil. Soc., 53, 764

Scheuer, P. A. G. 1974, MNRAS, 166, 329

Scott, S. E., et al. 2002, MNRAS, 331, 817

Stoyan, D., Kendall, W. S., \& Mecke, J. 1995, Stochastic Geometry and its Applications, 2nd ed. (Chichester: John Wiley \& Sons)

Stoyan, D., \& Stoyan, H. 1994, Fractals, Random Shapes and Point Fields (Chichester: John Wiley \& Sons)

Stuart, A., \& Ord, K. 1994, Kendall's Advanced Theory of Statistics, 6th ed. Vol. 1, Distribution Theory (London: Arnold)

Szapudi, I., \& Szalay, A. 1993, ApJ, 408, 43

Szapudi, I., Dalton, G. B., Efstathiou, G., \& Szalay, A. 1995, ApJ, 444,520

Szapudi, I., Colombi, S., \& Bernardeau, F. 1999, MNRAS, 310,428

Takeuchi, T. T., Hirashita, H., Ohta, K., Hattori, T. G., Ishii, T. T., \& Shibai, H. 1999, PASP, 111, 288

Takeuchi, T. T., Ishii, T. T., Hirashita, H., Yoshikawa, K, Matsuhara, H., Kawara, K., \& Okuda, H. 2001a, PASJ, 53, 37 Takeuchi, T. T., Kawabe, R., Kohno, K., Nakanishi, K., Ishii, T. T., Hirashita, H., \& Yoshikawa, K. 2001b, PASP, 113, 586

Takeuchi, T. T., Shibai, H., \& Ishii, T. T. 2002, Adv. Sp. Res., 30 2021

Toffolatti, L., Argüeso Gómez, F., De Zotti, G., Mazzei, P., Danese, L., \& Burigana, C. 1998, MNRAS, 297, 117

Totani, T., \& Takeuchi, T. T. 2002, ApJ, 570, 470

Vlad, M. O, Mackey, M. C., \& Ross, J. 1994, Phys. Rev. E, 50, 798

Wall, J. V. 1978, MNRAS, 182, 381

Wall, J. V., Scheuer, P. A. G., Pauliny-Toth, I. I. K., \& Witzel, A. 1982, MNRAS, 198, 221

Yano, T., \& Gouda, N. 1997, ApJ, 487, 473 